\title{
A lower bound for the rank of $J_{0}(q)$
}

by

E. Kowalski (Princeton, NJ) and P. Michel (Montpellier)

1. Introduction. Let $q$ be a prime number, and consider the abelian variety $J_{0}(q)$, the Jacobian of the modular curve $X_{0}(q)$. It is defined over $\mathbb{Q}$, of dimension $\operatorname{dim} J_{0}(q) \sim q / 12$. Eichler and Shimura [Sh] have shown that its Hasse-Weil $L$-function is given by

$$
L\left(J_{0}(q), s\right)=\prod_{f \in S_{2}(q)^{*}} L(f, s)
$$

where the product is over the finite set $S_{2}(q)^{*}\left(\left|S_{2}(q)^{*}\right|=\operatorname{dim} J_{0}(q)\right)$ of primitive weight 2 forms $f$ of level $q$, and the $L$-functions are normalized so that $\operatorname{Re}(s)=1 / 2$ is the critical line.

According to the Birch and Swinnerton-Dyer conjecture, one should have then

$$
\operatorname{rank} J_{0}(q)=\sum_{f \in S_{2}(q)^{*}} \operatorname{ord}_{s=1 / 2} L(f, s)
$$

and it is expected that

$$
\operatorname{rank} J_{0}(q) \sim \frac{1}{2} \operatorname{dim} J_{0}(q)
$$

based on heuristics concerning the zeros of $L$-functions.

In [KM1] we used the factorization (1) to obtain the upper bound

$$
\operatorname{rank} J_{0}(q) \leq C \operatorname{dim} J_{0}(q)
$$

for some absolute (and effectively computable, see [KM2]) constant $C>0$, on the Birch and Swinnerton-Dyer conjecture. This was proved by bounding from above the average order of vanishing of the $L$-functions at $s=1 / 2$.

Here we consider the dual problem of non-vanishing of $L(f, 1 / 2)$. More precisely we look at forms $f$ with order of vanishing exactly one. We prove

2000 Mathematics Subject Classification: Primary 11G40; Secondary 11F67. 
TheOREM 1. Let $\varepsilon>0$. For q large enough (in terms of $\varepsilon$ ), we have

$$
\left|\left\{f \in S_{2}(q)^{*} \mid L(f, 1 / 2)=0, L^{\prime}(f, 1 / 2) \neq 0\right\}\right| \geq\left(\frac{19}{54}-\varepsilon\right)\left|S_{2}(q)^{*}\right| .
$$

By work of Gross and Zagier [GZ], the product

$$
\prod_{f} L(f, s)
$$

over the forms $f$ with $L(f, 1 / 2)=0, L^{\prime}(f, 1 / 2) \neq 0$, is the $L$-function of a quotient of $J_{0}(q)$ with rank exactly equal to its dimension. Thus we have

Corollary 1. Let $\varepsilon>0$. For q large enough (in terms of $\varepsilon$ ), we have

$$
\operatorname{rank} J_{0}(q) \geq\left(\frac{19}{54}-\varepsilon\right) \operatorname{dim} J_{0}(q) .
$$

Since $19 / 54=0.35 \ldots$, this is quite close to the conjectured value.

The method used here works equally well for the non-vanishing of $L(f, 1 / 2)$ itself. We indicate briefly how they lead (more easily) to the

TheOREm 2. Let $\varepsilon>0$. For q large enough (in terms of $\varepsilon$ ), we have

$$
\left|\left\{f \in S_{2}(q)^{*} \mid L(f, 1 / 2) \neq 0\right\}\right| \geq\left(\frac{1}{6}-\varepsilon\right)\left|S_{2}(q)^{*}\right| .
$$

This result is weaker, however, than what Iwaniec and Sarnak [IS] have obtained in the course of their work on the Landau-Siegel zero. Indeed, their more advanced techniques can be used to improve the constant 19/54 to $7 / 16$.

Remarks. 1. Independently, and using different methods, VanderKam $[\mathrm{VdK}]$ has obtained the same non-vanishing results, except for a smaller numerical value of the proportion achieved.

2. Iwaniec, Luo and Sarnak [ILS] have proved (assuming the Generalized Riemann Hypothesis) that

$$
\sum_{f \in S_{2}(q)^{*}} \operatorname{ord}_{s=1 / 2} L(f, s) \leq(c+o(1))\left|S_{2}(q)^{*}\right|
$$

for some (explicit) $c<1$; this is of great significance for the conjectures and heuristics of Katz and Sarnak [KS].

We now give the precise statement of the main result.

Theorem 3. For any $0 \leq \Delta<1 / 4$ and any prime $q$ large enough (depending on $\Delta$ only), we have

$$
\sum_{\substack{f \in S_{2}(q)^{*} \\ L(f, 1 / 2)=0, L^{\prime}(f, 1 / 2) \neq 0}} 1 \geq \frac{1}{2}\left(1-\frac{1}{(1+2 \Delta)^{3}}\right) \operatorname{dim} J_{0}(q) .
$$

In particular, letting $\Delta \rightarrow 1 / 4$, Theorem 1 follows. 
Since the set of $f$ such that $L(f, s)$ has a simple zero at the critical point is contained in the set of odd forms, and both odd and even forms have asymptotic density $1 / 2$ among primitive forms, we have proved that for at least 70 percent of the odd forms, the order of $L(f, s)$ at the critical point is exactly one.

Remark. Coincidentally, Soundararajan [Sou], has shown that the proportion of quadratic twists of a given quadratic Dirichlet character $\chi$ for which $L(\chi \otimes \psi, 1 / 2) \neq 0$ satisfies the same lower bound, when the length of the mollifier is suitably parameterized. This is explained in part by the heuristics of Katz and Sarnak [KS]. Less clear is the coincidence of those proportions with that obtained by Conrey, Ghosh and Gonek [CGG] for the number of simple zeros of the Riemann $\xi$-function on the critical line.

Acknowledgments. This paper was begun at the Number Theory Conference in honor of Andrzej Schinzel, and we wish to take this opportunity to thank again the organizers for their efforts in making this an agreeable and successful meeting.

We also wish to thank H. Iwaniec and P. Sarnak for showing us some of their ongoing work [IS]. Also we thank the referee for carefully reading the most delicate parts of our arguments and pointing out some inaccuracies.

Notations. For any $q \geq 1$ we will write $\varepsilon_{q}$ for the trivial Dirichlet character modulo $q$. All summations over $f$ will be implicitly over $f \in$ $S_{2}(q)^{*}$, with other conditions explicitly indicated in the summation indices. We write $\log _{2} x:=\log \log x$.

Finally we make the following convention concerning the use of Vinogradov's and Landau's symbol $\ll, O($ ): the constants implied by these notations are meant to be absolute. In case there are other parameters involved, say $\varepsilon, \Delta$, we (usually) indicate the dependency of the constants by the subscript notations $\ll_{\varepsilon, \Delta}, O_{\varepsilon, \Delta}()$. The reader is encouraged to show good will towards analytic number theorists and interpret such inequalities in the most reasonable way (provided it is correct and proves the result which is sought...).

2. Non-vanishing in harmonic average. As in [KM1], we proceed by working first with the "harmonic" average

$$
\sum_{\substack{f \in S_{2}(q)^{*} \\ L(f, 1 / 2)=0, L^{\prime}(f, 1 / 2) \neq 0}}^{\mathrm{h}}
$$

where we write

$$
\sum_{f}^{\mathrm{h}} \alpha_{f}=\sum_{f} \frac{1}{4 \pi(f, f)} \alpha_{f}
$$


for any finite set $\alpha_{f}$ of complex numbers. We then derive the corresponding result for the "natural" average

$$
\sum_{\substack{f \in S_{2}(q)^{*} \\ L(f, 1 / 2)=0, L^{\prime}(f, 1 / 2) \neq 0}} 1 .
$$

2.1. The principle. As in previous investigations of such questions ([Du], $[\mathrm{Iw}],[\mathrm{KM} 1], \ldots)$, the theorem will follow, by an application of Cauchy's inequality, from a comparison of a lower bound for

$$
M_{1}:=\sum_{L(f, 1 / 2)=0}^{\mathrm{h}} M(f) L^{\prime}(f, 1 / 2)
$$

and an upper bound for

$$
M_{2}:=\sum_{L(f, 1 / 2)=0}^{\mathrm{h}}\left|M(f) L^{\prime}(f, 1 / 2)\right|^{2}
$$

for certain suitable complex numbers $M(f)$ (the "mollifier"). Indeed we have directly

so that

$$
M_{1} \leq\left(\sum_{L(f, 1 / 2)=0, L^{\prime}(f, 1 / 2) \neq 0}^{\mathrm{h}} 1\right)^{1 / 2} M_{2}^{1 / 2}
$$

$$
\sum_{L(f, 1 / 2)=0, L^{\prime}(f, 1 / 2) \neq 0}^{\mathrm{h}} 1 \geq M_{1}^{2} / M_{2} .
$$

We will follow this plan, except that in order to achieve the best possible numerical proportion, we will seek asymptotics for $M_{1}$ and $M_{2}$. It will be noticed that if the mollifier is ignored (take $M(f)=1$ ), a factor $\log q$ is lost in the final estimate.

In the case of the special values themselves, we consider of course

$$
N_{1}=\sum_{f \in S_{2}(q)^{*}}^{\mathrm{h}} M(f) L(f, 1 / 2), \quad N_{2}=\sum_{f \in S_{2}(q)^{*}}^{\mathrm{h}}|M(f) L(f, 1 / 2)|^{2}
$$

and compare.

2.2. The gamma factor effect. For $f \in S_{2}(q)^{*}$ we write its Fourier expansion

$$
f(z)=\sum_{n \geq 1} \lambda_{f}(n) n^{1 / 2} e(n z)
$$

and its $L$-function

$$
L(f, s)=\sum_{n \geq 1} \lambda_{f}(n) n^{-s}=\prod_{p}\left(1-\lambda_{f}(p) p^{-s}+\varepsilon_{q}(p) p^{-2 s}\right)^{-1}
$$

putting, as mentioned, the center of the critical strip at $1 / 2$. 
The functional equation is written in terms of the completed $L$-function

$$
\Lambda(f, s)=\left(\frac{\sqrt{q}}{2 \pi}\right)^{s} \Gamma(s+1 / 2) L(f, s),
$$

namely

$$
\Lambda(f, s)=\varepsilon_{f} \Lambda(f, 1-s)
$$

and the sign $\varepsilon_{f}$ of the functional equation is (see [Miy] for instance)

$$
\varepsilon_{f}=q^{1 / 2} \lambda_{f}(q)= \pm 1 .
$$

A form is said to be even (resp. odd) if $\varepsilon_{f}=1$ (resp. $\varepsilon_{f}=-1$ ). By the functional equation, this is the same parity as that of the order of $L(f, s)$ at $s=1 / 2$. We will write

$$
\varepsilon_{f}^{+}=\frac{1+\varepsilon_{f}}{2}, \quad \varepsilon_{f}^{-}=\frac{1-\varepsilon_{f}}{2}
$$

so $f \mapsto \varepsilon_{f}^{+} f$ is the projection of the space of primitive forms onto the space of even forms, and correspondingly for the odd ones. In particular, we have

$$
\left(\varepsilon_{f}^{ \pm}\right)^{2}=\varepsilon_{f}^{ \pm} .
$$

Since the gamma factor

$$
\gamma(s)=\left(\frac{\sqrt{q}}{2 \pi}\right)^{s} \Gamma(s+1 / 2)
$$

does not vanish at $1 / 2$, the order of $L(f, s)$ at $s=1 / 2$ is the same as that of $\Lambda(f, s)$. If $f$ is even, the vanishing of $\Lambda^{\prime}(f, 1 / 2)$ thus implies that

$$
L(f, 1 / 2)=0 \Rightarrow L^{\prime}(f, 1 / 2)=0 .
$$

From this we deduce an easy but very important proposition.

Proposition 1. Let $\left(\alpha_{f}\right)$ be any finite set of complex numbers. Then

$$
\sum_{L(f, 1 / 2)=0}^{\mathrm{h}} \alpha_{f} L^{\prime}(f, 1 / 2)=\sum_{f}^{\mathrm{h}} \varepsilon_{f}^{-} \alpha_{f} L^{\prime}(f, 1 / 2) .
$$

The point of this formula, which applies to the sums of type $M_{1}$ and $M_{2}$ above, is that an average over $f$ in the restricted subset where $L(f, 1 / 2)=0$ (the "non-rank 0" set) is written as an average over all $f$, for which suitable analytic summation formulae may apply, at the cost of inserting $\varepsilon_{f}$ which is much the same as $\lambda_{f}(q)$ (see (5)). We may notice at this point that this is special to the order 1 case: sums of the type

$$
\sum_{L(f, 1 / 2)=L^{\prime}(f, 1 / 2)=0}^{\mathrm{h}} \alpha_{f} L^{\prime \prime}(f, 1 / 2)
$$


- which one would like to study for estimating the (conjectural) dimension of the quotients of $J_{0}(q)$ of normalized rank 2- do not readily lend themselves to such an easy simplification.

2.3. Computing $M_{1}$. By Proposition 1, we have

$$
M_{1}=\sum_{f}^{\mathrm{h}} \varepsilon_{f}^{-} M(f) L^{\prime}(f, 1 / 2) .
$$

To make the sum manageable, we choose $M(f)$ of the shape

$$
M(f)=\sum_{m \leq M} x_{m} \lambda_{f}(m) m^{-1 / 2}
$$

for real numbers $\left(x_{m}\right)$ (and a parameter $M>0$ ) which we will try to choose to optimize the resulting bound (3). If $m>M$, we will write, for convenience, $x_{m}=0$. Now we only impose that the $x_{m}$ be supported on squarefree integers and satisfy

$$
x_{m} \ll(\tau(m)(\log q m))^{A}
$$

for some absolute constant $A>0$. We write $M=q^{\Delta}$, and will assume $0 \leq \Delta<1$.

First we express $L^{\prime}(f, 1 / 2)$ as a rapidly convergent series using contour integration and the functional equation: we consider the integral

$$
I=\frac{1}{2 i \pi} \int_{(2)} \Lambda(f, s+1 / 2) G(s) \frac{d s}{s^{2}}
$$

where $G$ is a polynomial of degree $N$ (large enough, $N=2$ works already) satisfying

$$
\begin{gathered}
G(-s)=G(s), \quad G(0)=1, \\
G(-N)=\ldots=G(-1)=0 .
\end{gathered}
$$

Notice that from the first of these, we also obtain

$$
G^{\prime}(0)=0, \quad G^{(3)}(0)=0 .
$$

If we shift the contour of integration to $\operatorname{Re}(s)=-1$ and apply the functional equation (4), we obtain

$$
2 \varepsilon_{f}^{-} I=\operatorname{Res}_{s=0} \frac{\Lambda(f, s+1 / 2) G(s)}{s^{2}} .
$$

If we use (11) and (9), this implies

$$
2 \varepsilon_{f}^{-} I=\Lambda^{\prime}(f, 1 / 2)
$$


whence, multiplying through by $\varepsilon_{f}^{-}$gives

$$
2 \varepsilon_{f}^{-} I=\varepsilon_{f}^{-}\left(\frac{\sqrt{q}}{2 \pi}\right)^{1 / 2} L^{\prime}(f, 1 / 2) .
$$

Expanding now $L(f, s)$ as a Dirichlet series in $I$ we get after some simplifications

$$
\varepsilon_{f}^{-} L^{\prime}(f, 1 / 2)=2 \varepsilon_{f}^{-} \sum_{l \geq 1} \lambda_{f}(l) l^{-1 / 2} V\left(\frac{2 \pi}{\sqrt{q}} l\right)
$$

with

$$
V(y)=\frac{1}{2 i \pi} \int_{(3 / 2)} \Gamma(s+1) G(s) y^{-s} \frac{d s}{s^{2}} .
$$

From this we obtain at once

$$
M_{1}=\sum_{l, m} x_{m}(l m)^{-1 / 2} V\left(\frac{2 \pi}{\sqrt{q}} l\right) \Delta_{-}(l, m)
$$

where

$$
\Delta_{-}(l, m)=2 \sum_{f}^{\mathrm{h}} \varepsilon_{f}^{-} \lambda_{f}(l) \lambda_{f}(m) .
$$

As can be expected, $\Delta_{-}$is a close relative to the Kronecker delta-symbol (in certain ranges).

Lemma 1. Let $\varepsilon>0$. Then for $l \geq 1$ and $1 \leq m \leq q$,

$$
\Delta_{-}(l, m)=\delta(l, m)+O\left(\frac{(l m)^{1 / 2+\varepsilon}}{q}\right)
$$

where $\delta$ is the Kronecker symbol.

Proof. By (5) we have

$$
\sum_{f}^{\mathrm{h}} \varepsilon_{f}^{-} \lambda_{f}(l) \lambda_{f}(m)=\frac{1}{2} \sum_{f}^{\mathrm{h}} \lambda_{f}(l) \lambda_{f}(m)+\frac{\sqrt{q}}{2} \sum_{f}^{\mathrm{h}} \lambda_{f}(q) \lambda_{f}(l) \lambda_{f}(m)
$$

and moreover $\lambda_{f}(q) \lambda_{f}(l)=\lambda_{f}(l q)$ for any $l$. We now apply Petersson's formula and classical bounds for Kloosterman sums and Bessel functions, supplemented in the second term by the remarks that for $m<q$ we have $l q \neq m$, and the Kloosterman sum $S(m, l q ; q)$ is a Ramanujan sum, from which a factor $q^{1 / 2}$ is saved when estimating sums $S(m, l q ; c q)$ for $(c, q)=1$, those for $q \mid c$ being easily treated. All this is explained in more detail in the next section, where a more refined analysis of the remainder term is required for the second moment. 
To conclude the analysis of $M_{1}$, we estimate $V$ (by shifting the contour to the left, or right):

$$
\begin{aligned}
& V(y)=-\log y-\gamma+O\left(y^{N}\right), \quad y \rightarrow 0, \\
& \forall j \geq 1, \quad V(y)=O_{j}\left(y^{-j}\right), \quad y \rightarrow \infty
\end{aligned}
$$

$\left(\gamma=-\Gamma^{\prime}(1)\right.$ being Euler's constant); then from (14), Lemma 1, and those estimates, we obtain the next proposition.

Proposition 2. Let $M=q^{\Delta}$ with $\Delta<1 / 2$, and define $\widehat{q}$ by

$$
\log \widehat{q}=-\log \frac{2 \pi}{\sqrt{q}}-\gamma .
$$

Then, for some absolute constant $c>0$,

$$
M_{1}=\sum_{m \leq M} \frac{x_{m}}{m} \log (\widehat{q} / m)+O\left(q^{-c}\right) .
$$

In the following, when we write an error term of the form $O\left(q^{-c}\right)$, it is implied that $c>0$, and the value of $c$ may change from line to line.

In the case of the first moment $N_{1}$ of special values, we consider similarly the integral

$$
\frac{1}{2 i \pi} \int_{(2)} \Lambda(f, s+1 / 2) G(s) \frac{d s}{s}
$$

and derive

$$
N_{1}=\sum_{m \leq M} \frac{x_{m}}{m}+O\left(q^{-c}\right)
$$

for some $c=c(\Delta)>0$ if $\Delta<1 / 2$. We only need the estimate

$$
\sum_{f \in S_{2}(q)^{*}}^{\mathrm{h}} \lambda_{f}(m) \lambda_{f}(n)=\delta(m, n)+O_{\varepsilon}\left((m n)^{1 / 2+\varepsilon} q^{-3 / 2}\right)
$$

(see below (23)).

2.4. Computing $M_{2}$. We now wish to get an expression for $M_{2}$ as a quadratic form in the $x_{m}$. A new phenomenon appears, however, at the point where we would like to appeal to Lemma 1, as the remainder term in the Petersson formula (the series of Kloosterman sums) cannot be ignored, and has to be analyzed to yield a contribution to the main term (compare e.g. $[\mathrm{DFI}])$.

2.4.1. Expressing $L^{\prime}(f, 1 / 2)^{2}$ for $f$ odd. We consider this time

$$
J=\frac{1}{2 i \pi} \int_{(2)} \Lambda(f, s+1 / 2)^{2} G(s) \frac{d s}{s^{3}}
$$


and proceed to evaluate it as before. From the formula

$$
L(f, s)^{2}=\zeta_{q}(2 s) \sum_{n \geq 1} \tau(n) \lambda_{f}(n) n^{-s}
$$

where $\zeta_{q}(s)=\zeta(s)\left(1-q^{-s}\right)$ is the Riemann zeta function with the Euler factor at $q$ removed, it follows that

$$
2 \cdot \frac{\sqrt{q}}{2 \pi} \sum_{n \geq 1} \lambda_{f}(n) \tau(n) n^{-1 / 2} W\left(\frac{4 \pi^{2} n}{q}\right)=\operatorname{Res}_{s=0} \frac{\Lambda(f, s+1 / 2)^{2} G(s)}{s^{3}}
$$

with

$$
W(y)=\frac{1}{2 i \pi} \int_{(1 / 2)} \zeta_{q}(1+2 s) \Gamma(s)^{2} G(s) y^{-s} \frac{d s}{s} .
$$

For our purpose, $W$ is basically a "cut-off" function. Indeed, we have the following

LEMma 2. The function $W$ satisfies

$$
\begin{gathered}
y^{i} W^{(j)}(y) \ll_{i, j}(\log (y+1 / y))^{3} \quad \text { for all } i \geq j \geq 0, \\
\forall j \geq 1, \quad W(y)=O_{j}\left(y^{-j}\right) .
\end{gathered}
$$

Moreover, there exists a polynomial $P$, independent of $q$, of degree at most 2 , such that for $y \rightarrow 0$

$$
W(y)=-\frac{1}{12}(\log y)^{3}+P(\log y)+O\left(q^{-1}(\log y)^{2}+y^{N}\right) .
$$

P r o of. The first two inequalities are obtained by the usual contour shifts and differentiating under the integral sign. As for the last, we write

$$
W(y)=\operatorname{Res}_{s=0} \frac{G(s) \Gamma(s)^{2} \zeta_{q}(1+2 s) y^{-s}}{s}+O\left(y^{N}\right)
$$

again by shifting, and simply compute the residue.

REMARK. The polynomial $P$ can be explicitly computed. However its exact value is of no importance in what follows, the only relevant fact being that its degree is $\leq 2$.

Now if $f$ is odd, we have $\Lambda(f, 1 / 2)=0$ and then we find that

$$
\left.\frac{d^{2}}{d s^{2}} \Lambda(f, s+1 / 2)^{2}\right|_{s=0}=2 \cdot \frac{\sqrt{q}}{2 \pi} L^{\prime}(f, 1 / 2)^{2}
$$

so, evaluating the residue, we derive for $f$ odd

$$
L^{\prime}(f, 1 / 2)^{2}=2 \sum_{n \geq 1} \lambda_{f}(n) \tau(n) n^{-1 / 2} W\left(\frac{4 \pi^{2} n}{q}\right) .
$$

2.4.2. Applying Petersson's formula. Working towards incorporating the mollifier, we fix some $0 \leq \Delta<1,1 \leq m \leq q^{\Delta}$, and consider the following 
average over $f$ :

$$
X(m)=\sum_{f \in S_{2}(q)^{*}}^{\mathrm{h}} \varepsilon_{f}^{-} \lambda_{f}(m) L^{\prime}(f, 1 / 2)^{2} .
$$

From (21) and (5), we have

$$
X(m)=\sum_{f}^{\mathrm{h}}\left(1-q^{1 / 2} \lambda_{f}(q)\right) \lambda_{f}(m) \sum_{n \geq 1} \tau(n) \lambda_{f}(n) n^{-1 / 2} W\left(\frac{4 \pi^{2} n}{q}\right) .
$$

For any $l_{1}$ and $l_{2}$, Petersson's formula is

$$
\sum_{f}^{\mathrm{h}} \lambda_{f}\left(l_{1}\right) \lambda_{f}\left(l_{2}\right)=\delta\left(l_{1}, l_{2}\right)-\mathcal{J}\left(l_{1}, l_{2}\right)
$$

where

$$
\mathcal{J}\left(l_{1}, l_{2}\right)=\frac{2 \pi}{q} \sum_{r \geq 1} r^{-1} S\left(l_{1}, l_{2} ; q r\right) J_{1}\left(\frac{4 \pi \sqrt{l_{1} l_{2}}}{q r}\right) .
$$

The trivial bound for this, from Weil's bound for Kloosterman sums and $J_{1}(x) \ll x$, is

$$
\mathcal{J}\left(l_{1}, l_{2}\right) \ll_{\varepsilon} \frac{\left(l_{1} l_{2}\right)^{1 / 2+\varepsilon}}{q^{3 / 2}} .
$$

Since $q$ is the level, $\lambda_{f}(q) \lambda_{f}(n)=\lambda_{f}(n q)$ for all $n$, and moreover $q n \neq m$ since $(m, q)=1$, therefore we get

$$
X(m)=X^{+}(m)+X^{-}(m)
$$

with

$$
\begin{gathered}
X^{+}(m)=\frac{\tau(m)}{\sqrt{m}} W\left(\frac{4 \pi^{2} m}{q}\right)-\sum_{n \geq 1} \frac{\tau(n)}{\sqrt{n}} W\left(\frac{4 \pi^{2} n}{q}\right) \mathcal{J}(n, m), \\
X^{-}(m)=q^{1 / 2} \sum_{n \geq 1} \frac{\tau(n)}{\sqrt{n}} W\left(\frac{4 \pi^{2} n}{q}\right) \mathcal{J}(q n, m) .
\end{gathered}
$$

2.4.3. Treatment of $X^{+}(m)$. If we use the trivial bound (23) and (19) ( $N=2$ is enough) the second term is seen to be

$$
\ll_{\varepsilon} m^{1 / 2+\varepsilon} q^{-1 / 2}(\log q)^{4}
$$

and by (20) we infer

(24) $X^{+}(m)=\frac{1}{12} \cdot \frac{\tau(m)}{\sqrt{m}}\left(\log \frac{\widehat{Q}}{m}\right)^{3}+\frac{\tau(m)}{\sqrt{m}} P\left(\log \frac{\widehat{Q}}{m}\right)+O_{\varepsilon}\left(\frac{m^{1 / 2} q^{\varepsilon}}{\sqrt{q}}\right)$,

with $\widehat{Q}$ defined by $\log \widehat{Q}=\log \left(q /\left(4 \pi^{2}\right)\right)$.

2.4.4. Treatment of $X^{-}(m)$. The contribution, in $\mathcal{J}(q n, m)$, of those $r$ for which $(r, q)>1$ (so $q \mid r)$ is also found to be $O\left((m n)^{1 / 2+\varepsilon} q^{-5 / 2}\right)$ and in 
toto this gives

$$
\ll_{\varepsilon} m^{1 / 2+\varepsilon} q^{-1}(\log q)^{4} .
$$

It remains to study

$$
\frac{2 \pi}{\sqrt{q}} \sum_{(r, q)=1} \frac{1}{r} \sum_{n \geq 1} \frac{\tau(n)}{\sqrt{n}} S(m, q n ; q r) J_{1}\left(\frac{4 \pi}{r} \sqrt{\frac{m n}{q}}\right) W\left(\frac{4 \pi^{2} n}{q}\right) .
$$

For $(r, q)=1$, the Kloosterman sum $S(m, q n ; q r)$ factorizes

$$
S(m, q n ; q r)=S(m \bar{q}, n ; r) S(0, m ; q)=-S(m \bar{q}, n ; r)
$$

since $S(0, m ; q)$ is a Ramanujan sum with $q$ prime, and $(m, q)=1$.

Fix $R>0$, to be chosen later (but such that $\log R \ll \log q$ ). In the previous expression we estimate the tail of the series for $r>R$ :

$$
\begin{array}{r}
-\frac{2 \pi}{\sqrt{q}} \sum_{\substack{r>R \\
(r, q)=1}} \frac{1}{r} \sum_{n \geq 1} \frac{\tau(n)}{\sqrt{n}} S(m \bar{q}, n ; r) J_{1}\left(\frac{4 \pi}{r} \sqrt{\frac{m n}{q}}\right) W\left(\frac{4 \pi^{2} n}{q}\right) \\
=O\left(\frac{m^{1 / 2+\varepsilon}(\log q)^{4}}{R^{1 / 2}}\right)
\end{array}
$$

and reduce the study of $X^{-}(m)$ to that of the remaining part, say $X^{\prime}(m)$.

2.4.5. Extraction of the main term. We denote by $X_{r}$ the inner sum in (the weighted) $X^{\prime}(m)$ :

$$
X_{r}=-\sum_{n \geq 1} \frac{\tau(n)}{\sqrt{n}} S(m \bar{q}, n ; r) J_{1}\left(\frac{4 \pi}{r} \sqrt{\frac{m n}{q}}\right) W\left(\frac{4 \pi^{2} n}{q}\right) \xi(n) .
$$

For technical reasons (which only occur because the weight is 2 ), we have fixed a $C^{\infty}$ function $\xi: \mathbb{R}^{+} \rightarrow[0,1]$ which satisfies

$$
\xi(x)=0, \quad 0 \leq x \leq 1 / 2, \quad \xi(x)=1, \quad x \geq 1
$$

and attached the weight $\xi(n)$ to the summation in $n$, without changing the value of $X_{r}$, of course.

Now we open the Kloosterman sum

$$
S(m \bar{q}, n ; r)=\sum_{d \bmod r}^{*} e\left(\frac{m \overline{q d}+n d}{r}\right)
$$

and take the summation over $d$ outside. For each $d$, Jutila's extension ([Jut], Theorem 1.7) of the Voronoï summation formula can be applied.

Proposition 3 (Jutila). Let $t: \mathbb{R}^{+} \rightarrow \mathbb{C}$ be a $C^{\infty}$ function which vanishes in a neighborhood of 0 and is rapidly decreasing at infinity. Then for $c \geq 1$ and $d$ coprime with $c$, we have 
This yields

$$
\begin{aligned}
\sum_{m \geq 1} \tau(m) e\left(\frac{d m}{c}\right) t(m)= & \frac{2}{c} \int_{0}^{\infty}\left(\log \frac{\sqrt{x}}{c}+\gamma\right) t(x) d x \\
& -\frac{2 \pi}{c} \sum_{h \geq 1} \tau(h) e\left(-\frac{\bar{d} h}{c}\right) \int_{0}^{\infty} Y_{0}\left(\frac{4 \pi \sqrt{h x}}{c}\right) t(x) d x \\
& +\frac{4}{c} \sum_{h \geq 1} \tau(h) e\left(\frac{\bar{d} h}{c}\right) \int_{0}^{\infty} K_{0}\left(\frac{4 \pi \sqrt{h x}}{c}\right) t(x) d x .
\end{aligned}
$$

$$
\begin{aligned}
X_{r}= & -\frac{2}{r} S(m, 0 ; r) \\
& \times \int_{0}^{\infty}\left(\log \frac{\sqrt{x}}{r}+\gamma\right) J_{1}\left(\frac{4 \pi}{r} \sqrt{\frac{m x}{q}}\right) W\left(\frac{4 \pi^{2} x}{q}\right) \xi(x) \frac{d x}{\sqrt{x}} \\
& +\frac{2 \pi}{r} \sum_{h \geq 1} \tau(h) S(h q-m, 0 ; r) \\
& \times \int_{0}^{\infty} Y_{0}\left(\frac{4 \pi \sqrt{h x}}{r}\right) J_{1}\left(\frac{4 \pi}{r} \sqrt{\frac{m x}{q}}\right) W\left(\frac{4 \pi^{2} x}{q}\right) \xi(x) \frac{d x}{\sqrt{x}} \\
& -\frac{4}{r} \sum_{h \geq 1} \tau(h) S(h q+m, 0 ; r) \\
& \times \int_{0}^{\infty} K_{0}\left(\frac{4 \pi \sqrt{h x}}{r}\right) J_{1}\left(\frac{4 \pi}{r} \sqrt{\frac{m x}{q}}\right) W\left(\frac{4 \pi^{2} x}{q}\right) \xi(x) \frac{d x}{\sqrt{x}} .
\end{aligned}
$$

We reserve for later consideration the last two sums (see Section 2.4.6), and proceed to immediately remove $\xi$ from the first, which we can do with an error which is at most

$$
\begin{aligned}
\frac{1}{\sqrt{q}} \sum_{r \leq R} \frac{1}{r^{2}}|S(m, 0 ; r)| \int_{0}^{1}\left|\left(\log \frac{\sqrt{x}}{r}+\gamma\right) J_{1}\left(\frac{4 \pi}{r} \sqrt{\frac{m x}{q}}\right) W\left(\frac{4 \pi^{2} x}{q}\right)\right| \frac{d x}{\sqrt{x}} \\
\ll \frac{1}{\sqrt{q}}(\log q)^{4}
\end{aligned}
$$

by (18) and simply $J_{1}(x) \ll 1$.

We are therefore studying

$$
\begin{aligned}
-\frac{4 \pi}{\sqrt{q}} \sum_{\substack{r \leq R \\
(r, q)=1}} \frac{1}{r^{2}} S(m, 0 ; r) \int_{0}^{\infty}\left(\log \frac{\sqrt{x}}{r}+\gamma\right) J_{1}\left(\frac{4 \pi}{r} \sqrt{\frac{m x}{q}}\right) W\left(\frac{4 \pi^{2} x}{q}\right) \frac{d x}{\sqrt{x}} \\
=-2 \sum_{\substack{r \leq R \\
(r, q)=1}} \frac{1}{r} S(m, 0 ; r) \int_{0}^{\infty}\left(\log \frac{\sqrt{q x}}{2 \pi}+\gamma\right) J_{1}(2 \sqrt{m x}) W\left(r^{2} x\right) \frac{d x}{\sqrt{x}}
\end{aligned}
$$

by the change of variable $x \mapsto\left(r^{2} q y\right) /\left(4 \pi^{2}\right)$. 
By (17), this is equal to

$$
\frac{1}{2 i \pi} \int_{(1 / 2)}(-2) Z_{m}^{R}(1+2 s) \zeta_{q}(1+2 s) s^{-1} \Gamma(s)^{2} G(s) L(s) d s,
$$

with

$$
\begin{aligned}
Z_{m}^{R}(s) & =\sum_{\substack{r \leq R \\
(r, q)=1}} S(m, 0 ; r) r^{-s}, \\
L(s) & =\int_{0}^{\infty}\left(\log \frac{\sqrt{q x}}{2 \pi}+\gamma\right) J_{1}(2 \sqrt{m x}) x^{-s-1 / 2} d x .
\end{aligned}
$$

Both $Z_{m}^{R}$ and $L$ can be computed.

Lemma 3. For $\operatorname{Re}(s)=\sigma>1$, we have

$$
Z_{m}^{R}(s)=\zeta_{q}(s)^{-1} \sum_{d \mid m} d^{1-s}+O_{\sigma}\left(\tau(m) R^{1-\sigma}\right) .
$$

Proof. By the formula giving the Ramanujan sum (the star meaning "prime to $q$ ")

$$
\begin{aligned}
Z_{m}^{R}(s) & =\sum_{r \leq R}^{*} r^{-s} \sum_{d \mid(m, r)} d \mu\left(\frac{r}{d}\right) \\
& =\sum_{d \mid m} d \sum_{f d \leq R}^{*} \mu(f)(f d)^{-s} \\
& =\sum_{d \mid m} d^{1-s}\left\{\zeta_{q}(s)^{-1}+O\left(\left(\frac{R}{d}\right)^{1-\sigma}\right)\right\} \\
& =\zeta_{q}(s)^{-1} \sum_{d \mid m} d^{1-s}+O\left(\tau(m) R^{1-\sigma}\right) .
\end{aligned}
$$

Lemma 4. Recall that $\log \widehat{Q}=\log \left(q /\left(4 \pi^{2}\right)\right)$. For all $s$ with $1 / 4<$ $\operatorname{Re}(s)<1$, we have

$$
L(s)=-\frac{1}{2} m^{s-1 / 2} \Gamma(-s) \Gamma(s)^{-1}\left(\log \frac{\widehat{Q}}{m}+2 \gamma+\psi(1+s)+\psi(1-s)\right)
$$

where $\psi=\Gamma^{\prime} / \Gamma$.

Proof. The following formula is valid for $-2<\operatorname{Re}(s)<-1 / 2$ (see $[\mathrm{GR}], 6.561 .14)$ :

$$
l(s):=\int_{0}^{\infty} J_{1}(x) x^{s} d x=2^{s} \Gamma\left(1+\frac{s}{2}\right) \Gamma\left(1-\frac{s}{2}\right)^{-1}
$$


and putting $y=2 \sqrt{m x}$ in $L(s)$ gives

$$
L(s)=4^{s} m^{s-1 / 2}\left(\left(\frac{1}{2} \log \frac{\widehat{Q}}{m}+\gamma\right) l(-2 s)+l^{\prime}(-2 s)\right) .
$$

From (31) we deduce

$$
l^{\prime}(s)=2^{s} \Gamma\left(1+\frac{s}{2}\right) \Gamma\left(1-\frac{s}{2}\right)^{-1}\left(\log 2+\frac{1}{2} \psi\left(1+\frac{s}{2}\right)+\frac{1}{2} \psi\left(1-\frac{s}{2}\right)\right)
$$

and the result follows.

This allows us to replace $Z_{m}^{R}(1+2 s)$ in (30) by $\sigma_{-2 s}(m) \zeta_{q}(1+2 s)^{-1}$, up to an error which is bounded by $O\left(\tau(m)(\log q) R^{-1}\right)$. Denote by $X^{\prime \prime}(m)$ the resulting expression.

The lemmas show that the integrand in $X^{\prime \prime}(m)$ is

$F(s)=m^{-1 / 2} s^{-1} G(s) \eta_{s}(m) \Gamma(s) \Gamma(-s)\left(\log \frac{\widehat{Q}}{m}+2 \gamma+\psi(1+s)+\psi(1-s)\right)$

where $\eta_{s}$ is the arithmetic function defined by

$$
\eta_{s}(m)=\sum_{a b=m}\left(\frac{a}{b}\right)^{s} .
$$

Thus, the integrand is seen to be an odd function of $s$, which is moreover holomorphic in the strip $|\operatorname{Re}(s)|<1$, except for a triple pole at $s=0$, and decreases exponentially in vertical strips. Shifting the contour to $\operatorname{Re}(s)=$ $-1 / 2$ and changing then $s$ into $-s$ allows us to conclude that

$$
X^{\prime \prime}(m)=\frac{1}{2} \operatorname{Res}_{s=0} F(s) .
$$

Around $s=0$, the following expansions hold:

$$
\begin{gathered}
\eta_{s}(m)=\tau(m)+\frac{1}{2} T(m) s^{2}+O\left(s^{3}\right), \\
G(s)=1+\frac{1}{2} G^{\prime \prime}(0) s^{2}+O\left(s^{3}\right), \\
s^{-1} \Gamma(s) \Gamma(-s)=-\frac{1}{s^{3}}+\frac{\gamma^{2}-\Gamma^{\prime \prime}(1)}{s}+O(s), \\
\log \frac{\widehat{Q}}{m}+2 \gamma+\psi(1+s)+\psi(1-s)=\log \frac{\widehat{Q}}{m}+\psi^{\prime \prime}(0) s^{2}+O\left(s^{4}\right),
\end{gathered}
$$

where $T$ is the arithmetic function defined by

$$
T(m)=\sum_{a b=m}\left(\log \frac{a}{b}\right)^{2} .
$$


Combining those, we obtain

$$
\frac{1}{2} \operatorname{Res}_{s=0} F(s)=-\frac{1}{4} \cdot \frac{T(m)}{\sqrt{m}}\left(\log \frac{\widehat{Q}}{m}\right)+\alpha \frac{\tau(m)}{\sqrt{m}}\left(\log \frac{\widehat{Q}}{m}\right),
$$

where we have set

$$
\alpha=\frac{1}{2}\left(\gamma^{2}-\Gamma^{\prime \prime}(1)-\frac{G^{\prime \prime}(0)}{2}-\psi^{\prime \prime}(0)\right) .
$$

If we now take $R=q^{2}$, we infer from (25), (26), and Lemmas 6 and 8 of Section 2.4.6 an approximate formula for $X^{-}(m)$.

Proposition 4. Let $0 \leq \Delta<1$ and $1 \leq m \leq q^{\Delta}$. For any $\varepsilon>0$

$$
\begin{aligned}
X^{-}(m)= & -\frac{1}{4} \cdot \frac{T(m)}{\sqrt{m}}\left(\log \frac{\widehat{Q}}{m}\right) \\
& +\alpha \frac{\tau(m)}{\sqrt{m}}\left(\log \frac{\widehat{Q}}{m}\right)+O_{\Delta, \varepsilon}\left(q^{\varepsilon}\left(\frac{m^{1 / 2}}{q}+\frac{1}{\sqrt{q}}\right)\right) .
\end{aligned}
$$

This together with (24) yields an approximate formula for $X(m)$.

Proposition 5. Set $P_{1}(X)=P(X)+\alpha X$. Then for $0 \leq \Delta<1 / 2$, and $1 \leq m \leq q^{\Delta}$, we have for any $\varepsilon>0$,

$$
\begin{aligned}
X(m)= & \frac{1}{12} \cdot \frac{\tau(m)}{\sqrt{m}}\left(\log \frac{\widehat{Q}}{m}\right)^{3}-\frac{1}{4} \cdot \frac{T(m)}{\sqrt{m}}\left(\log \frac{\widehat{Q}}{m}\right)+\frac{\tau(m)}{\sqrt{m}} P_{1}\left(\log \frac{\widehat{Q}}{m}\right) \\
& +O_{\Delta, \varepsilon}\left(\frac{m^{1 / 2} q^{\varepsilon}}{\sqrt{q}}\right) .
\end{aligned}
$$

For later use, we record a few properties of the function $T$.

Lemma 5. Let $\tau^{(i)}$ be defined for $i \geq 0$ by

$$
\tau^{(i)}(m)=\sum_{d \mid m}(\log d)^{i} .
$$

Then

$$
T(m)=4 \tau^{(2)}(m)-2(\log m) \tau^{(1)}(m) .
$$

Moreover, $T$ satisfies

$$
T\left(m_{1} m_{2}\right)=\tau\left(m_{1}\right) T\left(m_{2}\right)+\tau\left(m_{2}\right) T\left(m_{1}\right)
$$

for $\left(m_{1}, m_{2}\right)=1$.

Proof. The first formula is immediate, and the second follows from

$$
\sum_{a b=m}\left(\log \frac{a}{b}\right)=0 .
$$

2.4.6. Estimation of the integrals. We still have to vindicate our contention that the two expressions involving the Bessel functions $Y_{0}$ and $K_{0}$ 
in (28) and (29) are of smaller order of magnitude (in our situation) than the main term isolated in the previous section. We will denote by $Y(m)$ and $K(m)$ their respective contributions to $X(m)$.

Lemma 6. For all $\varepsilon>0$, we have

$$
K(m) \ll_{\varepsilon} \frac{q^{\varepsilon} m^{1 / 2}}{q} .
$$

Proof. Because $K_{0}$ has exponential decay at infinity and $\xi$ cuts off the small values of $x$, this is easy. We have

$$
K(m)=-\frac{8 \pi}{\sqrt{q}} \sum_{r \leq R}^{*} \frac{1}{r^{2}} \sum_{h \geq 1} \tau(h) S(h q+m, 0 ; r) k(h)
$$

(here and hereafter, the symbol $\sum^{*}$ restricts the summation to $r$ coprime with $q$ ) and $k(h)$ is the integral involving the $K_{0}$ function, for which we have (employing the bound $K_{0}(y) \ll y^{-1 / 2} e^{-y}$ )

$$
\begin{aligned}
k(h) & =\int_{0}^{\infty} K_{0}\left(\frac{4 \pi \sqrt{h x}}{r}\right) J_{1}\left(\frac{4 \pi}{r} \sqrt{\frac{m x}{q}}\right) W\left(\frac{4 \pi^{2} x}{q}\right) \xi(x) \frac{d x}{\sqrt{x}} \\
& =\frac{r}{2 \pi \sqrt{h}} \int_{0}^{\infty} K_{0}(y) J_{1}\left(\sqrt{\frac{m}{h q}} y\right) W\left(\frac{r^{2} y^{2}}{4 q h}\right) \xi\left(\frac{r^{2} y^{2}}{16 \pi^{2} h}\right) d y \\
& \ll \frac{r}{h} \sqrt{\frac{m}{q}}(\log q)^{3} \int_{\sqrt{h} r^{-1}}^{\infty} y^{1 / 2} e^{-y} d y \\
& \ll \frac{r}{h} \sqrt{\frac{m}{q}}(\log q)^{3} e^{-\sqrt{h} /(2 r)}
\end{aligned}
$$

so that

$$
\begin{aligned}
K(m) & \ll \frac{\sqrt{m}}{q}(\log q)^{3} \sum_{h \geq 1} \frac{\tau(h)}{h} e^{-\sqrt{h} /(2 R)} \sum_{r \leq R}^{*} \frac{(r, h q+m)}{r} \\
& \ll \frac{\sqrt{m}}{q}(\log q)^{4} \sum_{h \geq 1} \frac{\tau(h) \tau(h q+m)}{h} e^{-\sqrt{h} /(2 R)} \ll_{\varepsilon} \frac{q^{\varepsilon} \sqrt{m}}{q} .
\end{aligned}
$$

The case of $Y(m)$ is slightly more complicated because $Y_{0}$ is an oscillating function. We will use the following lemma which is quite standard.

LEMMA 7. Let $\nu \geq 0$ be a real number, and $J \geq 0$ an integer. If $f$ is a compactly supported $C^{\infty}$ function, and $\beta>0$ is a real number such that $f$ is supported on $[Y, 2 Y]$ and satisfies

$$
y^{j} f^{(j)}(y) \ll_{j}(1+\beta Y)^{j}
$$


for $0 \leq j \leq J$, then for any $\alpha>1$,

$$
\int_{0}^{\infty} Y_{\nu}(\alpha y) f(y) d y \ll\left(\frac{1+\beta Y}{1+\alpha Y}\right)^{J} Y
$$

Proof. One could write the asymptotic development of $Y_{0}$ to show the oscillating behavior and integrate by parts, but it is cleaner (and amounts to the same thing) to make use of the recurrence formula

$$
\left(y^{\nu} Y_{\nu}(y)\right)^{\prime}=y^{\nu} Y_{\nu-1}(y)
$$

to get, integrating by part also,

$$
\int_{0}^{\infty} Y_{\nu}(\alpha y) f(y) d y=\frac{1}{\alpha} \int_{0}^{\infty} Y_{\nu+1}(\alpha y)\left(-f^{\prime}(y)+(\nu+1) \frac{f(y)}{y}\right) d y .
$$

Let $g(y)=-f^{\prime}(y)+(\nu+1) f(y) / y$; it is immediate that $g$ satisfies

$$
y^{j+1} g^{(j)}(y) \ll(1+\beta Y)^{j+1} \quad \text { for } 0 \leq j \leq J-1
$$

so that by iterating this procedure we obtain

$$
\int_{0}^{\infty} Y_{\nu}(\alpha y) f(y) d y=\frac{1}{\alpha^{J}} \int_{0}^{\infty} Y_{\nu+J}(\alpha y) h(y) d y
$$

where the function $h$ is such that

$$
y^{J} h(y) \ll_{J}(1+\beta Y)^{J}
$$

and therefore the result follows by using $Y_{\nu+J}(y) \ll_{J+\nu} 1$.

LEMMA 8. For $\Delta<1, m \leq q^{\Delta}$, and any $\varepsilon>0$, we have

$$
Y(m) \ll_{\Delta, \varepsilon} q^{\varepsilon}\left(\frac{m^{1 / 2}}{q}+\frac{1}{\sqrt{q}}\right) .
$$

Proof. We write

$$
Y(m)=\frac{4 \pi^{2}}{\sqrt{q}} \sum_{r \leq R}^{*} \frac{1}{r^{2}} \sum_{h} \tau(h) S(h q-m, 0 ; r) y(h)
$$

with

$$
y(h)=\int_{0}^{\infty} Y_{0}\left(\frac{4 \pi \sqrt{h x}}{r}\right) J_{1}\left(\frac{4 \pi}{r} \sqrt{\frac{m x}{q}}\right) W\left(\frac{4 \pi^{2} x}{q}\right) \xi(x) \frac{d x}{\sqrt{x}} .
$$

Note that $h q \neq m$ since $m<q$, so the Ramanujan sum never degenerates to the trivial sum $S(0,0 ; r)=r-1$ but is always much smaller.

We make a smooth dyadic partition of unity, so $\xi=\sum_{k>1} \xi_{k}$ where each $\xi_{k}$ is a $C^{\infty}$ function with compact support in a dyadic interval $\left[X_{k}, 2 X_{k}\right]$ that satisfies

$$
x^{j} \xi_{k}^{(j)}(x) \ll 1 \quad \text { for all } j \geq 0,
$$


the implied constants depending on $j$ alone (in particular, they are uniform in $k$ ).

We study each $\xi_{k}$ individually, but we keep writing $\xi$ instead of $\xi_{k}$, and accordingly we use $X$ rather than $X_{k}$.

By the change of variable $2 r^{-1} \sqrt{x}=y$, the integral is

$$
y(h)=r \int_{0}^{\infty} Y_{0}(2 \pi \sqrt{h} x) J_{1}\left(2 \pi \sqrt{\frac{m}{q}} x\right) W\left(\frac{\pi^{2} r^{2} x^{2}}{q}\right) \xi\left(\frac{r^{2} x^{2}}{4}\right) d x,
$$

so we define the function $f$ by

$$
f(x)=J_{1}\left(2 \pi \sqrt{\frac{m}{q}} x\right) W\left(\frac{\pi^{2} r^{2} x^{2}}{q}\right) \xi\left(\frac{r^{2} x^{2}}{4}\right) .
$$

This is a $C^{\infty}$ function compactly supported in the dyadic interval $[\varrho, 2 \varrho]$, with

$$
\varrho=2 \frac{\sqrt{X}}{r}
$$

We first treat the case

$$
1 / 2 \leq X \leq q^{2}
$$

(which involves $\ll \log q$ terms) and for this quote from (18) the bound

$$
x^{j} W^{(j)}(x) \ll_{j}(\log q)^{3} \quad \text { for all } j \geq 0,
$$

valid for $1 / q \ll x \ll 2 q^{2}$. This, together with (35), the recurrence relation $\left(x^{\nu} J_{\nu}(x)\right)^{\prime}=x^{\nu} J_{\nu-1}(x)$ and some elementary manipulations with inequalities, yields

$$
x^{j} f^{(j)}(x) \ll_{j}\left(1+\sqrt{\frac{m}{q}} x\right)^{j}(\log q)^{3} \quad \text { for all } j \geq 0 .
$$

Thus, we are in a position to apply the preceding lemma to $f$ with $\alpha=2 \pi \sqrt{h}, \beta=2 \pi \sqrt{m / q}$ and $Y=\varrho$. Unfortunately, this is inefficient for certain ranges of $X, r$ and/or $h$, and it will be necessary to split into other cases.

What the lemma implies is, for any integer $J \geq 0$,

$$
y(h) \ll_{J} r \frac{(1+\sqrt{m / q} \varrho)^{J}}{(1+\sqrt{h} \varrho)^{J}}(\log q)^{3} .
$$

Consider first the case $\varrho>2$, or $r<\sqrt{X}$ : applying (39) with $J \geq 3$ (to win convergence in $h$ ) yields a contribution in (34) which is therefore 


$$
\begin{aligned}
& \ll_{J} \frac{(\log q)^{3}}{\sqrt{q}} \sum_{r<\sqrt{X}}^{*} \frac{1}{r^{2}} r \varrho^{-(J-1)}\left(1+\sqrt{\frac{m}{q}} \varrho\right)^{J} \tau(r) \\
& \ll_{J} \frac{(\log q)^{3}}{\sqrt{q}}\left(\sum_{r<\sqrt{m X / q}}^{*} \varrho \frac{\tau(r)}{r}\left(\sqrt{\frac{m}{q}}\right)^{J}+\sum_{\sqrt{m X / q} \leq r<\sqrt{X}}^{*} \frac{\tau(r)}{r}\right) \\
& \ll_{J} \frac{(\log q)^{5+J}}{\sqrt{q}}\left(1+q^{1+J(\Delta-1) / 2}\right), \quad \text { since } m / q \leq q^{\Delta-1},
\end{aligned}
$$

at which point, since $\Delta<1$, we can choose $J$ large enough so that $1+J(\Delta-1) / 2 \leq 0$ to conclude that this part is

$$
\ll_{\Delta, \varepsilon} q^{\varepsilon} / \sqrt{q}
$$

(in this argument, the reader should keep in mind that $h q \neq m$ since $m<q$ ).

On the other hand, for $\varrho \leq 1$, we split the summation in $h$ in the following way:

$$
\sum_{h \geq 1}=\sum_{h \leq \varrho^{-2(1+\kappa)}}+\sum_{h>\varrho^{-2(1+\kappa)}}
$$

where $\kappa>0$ will be chosen (sufficiently small) a little later.

For the first sum, we come back to (35), using again $J_{1}(x) \ll x, Y_{0}(x) \ll$ $1+|\log x|$ to derive first the bound

$$
y(h) \ll \sqrt{\frac{m}{q}} \frac{X}{r}(\log q)^{3} .
$$

Then, since $|S(h q-m, 0 ; r)| \leq \sum_{d \mid(h q-m, r)} d$, we have

$$
\begin{aligned}
\frac{4 \pi^{2}}{\sqrt{q}} \sum_{\sqrt{X} \leq r \leq R}^{*} \frac{1}{r^{2}} \sum_{h \leq \varrho^{-2(1+\kappa)}} \tau(h) S(h q-m, 0 ; r) y(h) \\
\quad \ll \frac{(\log q)^{3}}{\sqrt{q}} \sum_{\sqrt{X} \leq r \leq R}^{*} \frac{1}{r^{2}} \sum_{h \leq \varrho^{-2(1+\kappa)}} \tau(h) \sum_{d \mid(h q-m, r)} d \frac{X}{r} \sqrt{\frac{m}{q}} \\
\quad \ll \frac{\sqrt{m}}{q}(\log q)^{3} X \sum_{h \leq\left(R^{2} / X\right)^{1+\kappa}} \tau(h) \sum_{\sqrt{X} h^{\theta} \leq r \leq R}^{*} \sum_{d \mid(h q-m, r)} \frac{d}{r^{3}}
\end{aligned}
$$

(exchanging the order of summation), where $\theta=(2+2 \kappa)^{-1}$. We transform the inner sum over $d$ and $r$ and estimate

$$
\begin{aligned}
\sum_{d \mid h q-m} \frac{1}{d^{2}} \sum_{\sqrt{X} h^{\theta} \leq d r \leq R}^{*} \frac{1}{r^{3}} & \ll X^{-1} h^{-2 \theta} \sum_{d \mid h q-m} 1 \\
& \ll \tau(h q-m) X^{-1} h^{-1+\kappa /(1+\kappa)} .
\end{aligned}
$$


Then (41) is estimated to be

$$
\begin{aligned}
& \ll \frac{\sqrt{m}}{q}(\log q)^{3} X \sum_{h \leq\left(R^{2} / X\right)^{1+\kappa}} \tau(h) \sum_{d \mid h q-m} \frac{1}{d^{2}} \sum_{\sqrt{X} h^{\theta} \leq d r \leq R}^{*} \frac{1}{r^{3}} \\
& \ll \frac{\sqrt{m}}{q}(\log q)^{3} X \sum_{h \leq\left(R^{2} / X\right)^{1+\kappa}} \tau(h) \tau(h q-m) X^{-1} h^{-1+\kappa /(1+\kappa)} \\
& \ll \varepsilon \frac{\sqrt{m}}{q} q^{\varepsilon} R^{2 \kappa} \quad \text { for all } \varepsilon>0 .
\end{aligned}
$$

For the second sum, applying (39) for $J \geq 3$ entails

and so as above

$$
y(h) \ll_{J} \sqrt{X} \varrho^{-J} h^{-J / 2}(\log q)^{3}
$$

$$
\begin{aligned}
\frac{4 \pi^{2}}{\sqrt{q}} \sum_{\sqrt{X} \leq r \leq R}^{*} \frac{1}{r^{2}} \sum_{h>\varrho^{-2(1+\kappa)}} \tau(h) S(h q-m, 0 ; r) y(h) \\
\ll_{J} \frac{\sqrt{X}}{\sqrt{q}}(\log q)^{3} \sum_{\sqrt{X} \leq r \leq R}^{*} \frac{1}{r^{2}} \sum_{h>\varrho^{-2(1+\kappa)}} \tau(h) \sum_{d \mid(h q-m, r)} d \varrho^{-J} h^{-J / 2} \\
\ll_{J} \frac{X^{(1-J) / 2}}{\sqrt{q}}(\log q)^{3} \sum_{h \geq 1} \tau(h) h^{-J / 2} \sum_{d \mid h q-m} d^{J-1} \sum_{r d \leq \sqrt{X} h^{\theta}} r^{J-2}
\end{aligned}
$$

(where $\theta=(2+2 \kappa)^{-1}$ as before)

$$
\ll_{J} \frac{(\log q)^{3}}{\sqrt{q}} \sum_{h \geq 1} \tau(h) \tau(h q-m) h^{-J / 2+\theta(J-1)} .
$$

We choose $\kappa=\varepsilon / 4$, then $J$ large enough so that $J(\theta-1 / 2)-\theta>1$ (in addition to the previous condition that $1+J(\Delta-1) / 2 \leq 0)$, so that the series over $h$ in (43) converges absolutely. Then (42) and (43) together are

$$
\ll_{\Delta, \varepsilon} q^{\varepsilon}\left(\frac{m^{1 / 2}}{q}+\frac{1}{\sqrt{q}}\right) .
$$

Finally, we return to the case $X>q^{2}$ which remains. We appeal to (19) (for $j=2$ ), and again use elementary estimations to prove that for $X>q$ the function $f$ satisfies the better bound

$$
x^{j} f^{(j)}(x) \ll\left(1+x \sqrt{\frac{m}{q}}\right)^{j} q^{2}(r x)^{-4} .
$$

The lemma then admits an immediate generalization to the effect that

$$
y(h) \ll r \varrho \frac{(1+\sqrt{m / q} \varrho)^{J}}{(1+\sqrt{h} \varrho)^{J}} \cdot \frac{q^{2}}{X^{2}}(\log q)^{3}
$$

in addition to the bound in (39). 
Since $X>q^{2}$, the quantity saved is $q^{2} / X^{2} \ll X^{-1}$ which is more than sufficient to allow for the sum over the dyadic values of $X$ involved to converge, and proves that all the previous bounds where (39) was used remain valid. The only place where this is not the case is the inequality (42), but this part of the sum is void for $\sqrt{X}>R$ and the former estimate works in the larger interval $X \leq R^{2}$.

2.4.7. A formula for the second moment. The definition of $M_{2}$ yields

$$
M_{2}=\sum_{b} \frac{1}{b} \sum_{m_{1}, m_{2} \leq M} \frac{X\left(m_{1} m_{2}\right)}{\sqrt{m_{1} m_{2}}} x_{b m_{1}} x_{b m_{2}} .
$$

Proposition 6. Assume $M=q^{\Delta}$ with $\Delta<1 / 4$. Then there exists $c>0$ such that

$$
M_{2}=\frac{1}{12} M_{21}-\frac{1}{4} M_{22}+M_{3}+O\left(q^{-c}\right)
$$

where

$$
\begin{aligned}
M_{21} & =\sum_{b} \frac{1}{b} \sum_{m_{1}, m_{2}} \frac{\tau\left(m_{1} m_{2}\right)}{m_{1} m_{2}} x_{b m_{1}} x_{b m_{2}}\left(\log \frac{\widehat{Q}}{m_{1} m_{2}}\right)^{3}, \\
M_{22} & =\sum_{b} \frac{1}{b} \sum_{m_{1}, m_{2}} \frac{T\left(m_{1} m_{2}\right)}{m_{1} m_{2}} x_{b m_{1}} x_{b m_{2}}\left(\log \frac{\widehat{Q}}{m_{1} m_{2}}\right) \\
M_{3} & =\sum_{b} \frac{1}{b} \sum_{m_{1}, m_{2}} \frac{\tau\left(m_{1} m_{2}\right)}{m_{1} m_{2}} P_{1}\left(\log \frac{\widehat{Q}}{m_{1} m_{2}}\right) .
\end{aligned}
$$

Proof. We apply Proposition 5 , with $R=q$, to evaluate $X\left(m_{1} m_{2}\right)$ in (44). The first three terms give exactly the three quadratic forms $M_{21}$, $M_{22}$ and $M_{3}$. Moreover, by (8), the error term is dominated, for any $\varepsilon>0$, by

$$
q^{-1 / 2+\varepsilon}\left(\sum_{m \leq M}\left|x_{m}\right|\right)^{2} \ll M^{2} q^{-1 / 2+2 \varepsilon} .
$$

If $\Delta<1 / 4$, we can take $\varepsilon$ small enough so that this is $O\left(q^{-c}\right)$ for some $c>0$.

Our strategy is now to write $M_{21}$ as a linear combination of easily diagonalized quadratic forms; the simplest in shape, say $\Pi$, is chosen and we are able to select $x_{m}$ to optimize the value of $\Pi$ with respect to $M_{1}$. Then the remaining terms in $M_{21}$ are evaluated, and so is $M_{22}$. Both are of the same order of magnitude, so our choice may not be perfectly optimal. On the other hand, with our specific choice of $x_{m}$, we finally prove that $M_{3}$ gives a smaller contribution, namely that

$$
M_{3}=O\left(M_{21} / \log q\right) \text {. }
$$


The second moment $N_{2}$ of central values $L(f, 1 / 2)$ is much simpler to handle: no detailed analysis of the remainder term in the Petersson formula is needed, (23) being sufficient to evaluate $N_{2}$ asymptotically for $M=q^{\Delta}$, $\Delta<1 / 4$. This is because the sign of the functional equation is always +1 for $L(f, s)^{2}$ and no contamination by $\varepsilon_{f}^{-}$occurs. Considering the integral

$$
\frac{1}{2 i \pi} \int_{(2)} L(f, s+1 / 2)^{2} G(s)^{2} \frac{d s}{s}
$$

one finds that

$$
L(f, 1 / 2)^{2}=2 \sum_{n \geq 1} \frac{\lambda_{f}(n)}{\sqrt{n}} \tau(n) U\left(\frac{4 \pi^{2} n}{q}\right)
$$

with

$$
U(y)=2 \frac{1}{2 i \pi} \int_{(2)} \zeta_{q}(1+2 s) G(s)^{2} \Gamma(s+1)^{2} y^{-s} \frac{d s}{s} .
$$

This test function decays faster than any polynomial as $y \rightarrow \infty$ and satisfies

$$
U(y)=-\frac{\varphi(q)}{q} \log y+C_{q}+O\left(y^{1 / 2}\right)
$$

as $y \rightarrow 0$. Here $C_{q}=c_{0}+O\left(q^{-1} \log q\right)$ for some explicitly computable, but unimportant, absolute constant $c_{0}$. Then computations similar to that leading to the main term in $M_{2}$ (but simpler) yield the expression

$$
N_{2}=\sum_{b} \frac{1}{b} \sum_{m_{1}, m_{2}} \frac{\tau\left(m_{1} m_{2}\right)}{m_{1} m_{2}} x_{b m_{1}} x_{b m_{2}}\left(\log \frac{\widehat{Q}}{m_{1} m_{2}}\right)+O\left(q^{-c}\right)
$$

for some $c=c(\Delta)>0$, for $\Delta<1 / 4$.

The optimization of $N_{2}$ proceeds in a way similar to that of $M_{2}$. We let $N_{21}$ denote the quadratic form which is the main term of $N_{2}$.

2.5. The preferred quadratic form $I$. Separating $m_{1}$ and $m_{2}$ in (46) by means of the formula

$$
\tau\left(m_{1} m_{2}\right)=\sum_{a \mid\left(m_{1}, m_{2}\right)} \mu(a) \tau\left(\frac{m_{1}}{a}\right) \tau\left(\frac{m_{2}}{a}\right)
$$

we get

(51) $\quad M_{21}=\sum_{b} \frac{1}{b} \sum_{a} \frac{\mu(a)}{a^{2}} \sum_{m_{1}, m_{2}} \frac{\tau\left(m_{1}\right) \tau\left(m_{2}\right)}{m_{1} m_{2}} x_{a b m_{1}} x_{a b m_{2}}\left(\log \frac{\widehat{Q}}{a^{2} m_{1} m_{2}}\right)^{3}$.

We define the following arithmetic functions:

$$
\nu_{t}(k)=\frac{1}{k} \sum_{a b=k} \frac{\mu(a)(\log a)^{t}}{a} \quad \text { for } t=0,1,2,3 ;
$$




$$
h(m)=\frac{\tau(m)}{m} .
$$

Then expanding the logarithm in (51) and rearranging, we see that $M_{21}$ is a linear combination of the quadratic forms $\Pi(t, u, v, w)$ in the $x_{m}$ 's defined by

$$
\begin{aligned}
& \Pi(t, u, v, w) \\
& =(\log \widehat{Q})^{u} \sum_{k} \nu_{t}(k) \sum_{m_{1}, m_{2}} h\left(m_{1}\right) h\left(m_{2}\right)\left(\log m_{1}\right)^{v}\left(\log m_{2}\right)^{w} x_{k m_{1}} x_{k m_{2}}
\end{aligned}
$$

where $t, u, v$ and $w$ are non-negative integers such that $t+u+v+w=3\left({ }^{1}\right)$.

We further restrict our attention to $\Pi(u, v, w):=\Pi(0, u, v, w)$; again it will be seen that for the chosen $\left(x_{m}\right)$,

$$
\Pi(t, u, v, w)=O\left(\Pi(0, u, v, w) \frac{\left(\log _{2} q\right)^{t}}{\log q}\right)
$$

which justifies this restriction. Accordingly we write $\nu$ for $\nu_{0}$, for which we have the formula

$$
\nu(k)=\varphi(k) / k^{2} \quad \text { for } k \leq M .
$$

The part of the expansion of $M_{21}$ involving those $\Pi(u, v, w)$ is then (using the obvious symmetry $\Pi(u, v, w)=\Pi(u, w, v))$ denoted by $m_{21}$ :

$$
\begin{aligned}
m_{21}= & \Pi(3,0,0)-6 \Pi(2,1,0)+6 \Pi(1,1,1)+6 \Pi(1,2,0) \\
& -6 \Pi(0,1,2)-2 \Pi(0,0,3) .
\end{aligned}
$$

Finally, we choose the one quadratic form $\Pi:=\Pi(3,0,0)$ as reference: we will choose $\left(x_{m}\right)$ to optimize $\Pi$ and evaluate afterwards the other $\Pi(u, v, w)$, for this choice, before doing the same with $M_{22}$.

Similarly, for $N_{2}$, by (50) we have

$$
N_{21}=\Pi(1,0,0)-2 \Pi(0,1,0)+2 \Pi(1,0,0,0)
$$

(and the last term will be of smaller order of magnitude).

2.5.1. Optimizing $\Pi$. Making the linear change of variable

$$
y_{k}=\sum_{m} h(m) x_{k m}
$$

we have the immediate diagonalization

$$
\Pi=(\log \widehat{Q})^{3} \sum_{k} \nu(k) y_{k}^{2} .
$$

$\left({ }^{1}\right)$ Actually, $M_{3}$ is also such a linear combination with the difference that $t+u+v+$ $w \leq 2$. This will explain (49). 
Conversely, let $g$ be the Dirichlet convolution inverse of $h$, then

$$
x_{m}=\sum_{k} g(k) y_{k m} .
$$

From this we express the linear form $\left(^{2}\right)$ in (15) in terms of $y_{k}$

$$
M_{1}=\sum_{m} \frac{x_{m}}{m} \log \frac{\widehat{q}}{m}=\sum_{k} j(k) y_{k}
$$

where

$$
j(k)=\sum_{a b=k} g(a) \frac{\log (\widehat{q} / b)}{b} .
$$

LEMma 9. For any integer $k \geq 1$ we have

$$
j(k)=\frac{\mu(k)}{k}(\log \widehat{q} k) .
$$

Proof. We have $\sum_{k \geq 1} g(k) k^{-s}=\zeta(s+1)^{-2}$ and therefore

$$
\begin{aligned}
\sum_{k \geq 1} j(k) k^{-s} & =\zeta(s+1)^{-2}\left((\log \widehat{q}) \zeta(s+1)+\zeta^{\prime}(s+1)\right) \\
& =(\log \widehat{q}) \zeta(s+1)^{-1}-\left(\zeta^{-1}\right)^{\prime}(s+1)
\end{aligned}
$$

whence the result.

By Cauchy's inequality, the best choice to optimize $\Pi$ with respect to $M_{1}$ is

$$
y_{k}= \begin{cases}\frac{j(k)}{\nu(k)}=\frac{k \mu(k)}{\varphi(k)}(\log \widehat{q} k) & \text { if } k \leq M, \\ 0 & \text { if } k>M,\end{cases}
$$

and $x_{m}$ is given by (60), from which (and the lemma) the conditions required in Section 2.3 are immediately verified.

We now compute the various terms in (57) in order to apply the CauchySchwarz inequality $(3)\left({ }^{3}\right)$.

LEMMA 10. With the previous notations and hypothesis, with $M=q^{\Delta}$, we have

$$
\begin{gathered}
M_{1}=(\log q)^{3} \Delta\left(\frac{\Delta^{2}}{3}+\frac{\Delta}{2}+\frac{1}{4}\right)+O\left((\log q)^{2}\right), \\
\Pi=(\log q)^{6} \Delta\left(\frac{\Delta^{2}}{3}+\frac{\Delta}{2}+\frac{1}{4}\right)+O\left((\log q)^{5}\right) .
\end{gathered}
$$

$\left({ }^{2}\right)$ Strictly speaking, the main term of the linear form, but we will keep the same notation.

$\left({ }^{3}\right)$ Since $j(k)$ is about $(\log k) / k$ and $\nu$ is about $k^{-1}$, it is already quite clear that we will get a positive (harmonic) proportion if $M=q^{\Delta}$ with $\Delta>0$. 
Proof. By the choice of $\left(y_{k}\right)$, we have

$$
(\log \widehat{Q})^{-3} \Pi=M_{1}=\sum_{k} \frac{j(k)^{2}}{\nu(k)}=\sum_{k} \frac{\mu(k)^{2}}{\varphi(k)}(\log \widehat{q} k)
$$

whence the result follows, by partial summation, from

$$
\sum_{k \leq K} \frac{\mu(k)^{2}}{\varphi(k)}=\log K+O(1)
$$

2.5.2. Estimation of $\Pi(u, v, w)$. For the other quadratic forms, we write $\Pi(u, v, w)=(\log \widehat{Q})^{u} \sum_{k} \nu(k) y_{k}^{(v)} y_{k}^{(w)} \quad$ where $\quad y_{k}^{(i)}=\sum_{m} h(m)(\log m)^{i} x_{k m}$.

We can express $y_{k}^{(i)}$ in terms of $\left(y_{k}\right)$ using the higher von Mangoldt function $\Lambda_{i}$, which is defined by the Dirichlet convolution

$$
\Lambda_{i}=\mu *(\log )^{i},
$$

so that $(\log m)^{i}=\sum_{a b=m} \Lambda_{i}(a)$. From this, and the fact that the $x_{m}$ 's are supported on squarefree integers, we derive

$$
y_{k}^{(i)}=\sum_{l \leq M / k} h(l) \Lambda_{i}(l) y_{k l} .
$$

We state the properties of $\Lambda_{i}$ which we will use.

- $\Lambda_{1}=\Lambda$, the usual von Mangoldt function.

- $\Lambda_{i}$ is supported on integers having at most $i$ distinct prime factors.

- If $m=p_{1} \ldots p_{i}$, for distinct primes $p_{1}, \ldots, p_{i}$, then

$$
\Lambda_{i}(m)=i !\left(\log p_{1}\right) \ldots\left(\log p_{i}\right) .
$$

- If $p_{1}$ and $p_{2}$ are distinct primes, then

$$
\Lambda_{i}\left(p_{1}\right)=\left(\log p_{1}\right)^{i}, \quad \Lambda_{3}\left(p_{1} p_{2}\right)=3\left(\log p_{1}\right)\left(\log p_{2}\right)\left(\log p_{1} p_{2}\right) .
$$

All of these are well known and (or) easy to prove from the recurrence relation

$$
\Lambda_{i+1}=(\log ) \Lambda_{i}+\Lambda * \Lambda_{i} .
$$

In (63) we are thus actually dealing with a sum over squarefree $l$ having at most $i$ prime factors, and $i \leq 3$. We separate the sum into the parts with a fixed number of prime factors, which produces multiple (at most triple) sums over primes (of Mertens type since $h(l)=2^{j} l^{-1}$ for such $l$ with $\omega(l)=j$ prime factors). 
The subsum with $i$ distinct prime factors is, by the above,

$$
\begin{aligned}
2^{i} i ! \sum_{\substack{l \leq M / k \\
\omega(l)=i}} & \frac{\Lambda_{i}(l)}{l} \mu(k l)(\log \widehat{q} k l) \frac{k l}{\varphi(k l)} \\
= & (-2)^{i} i ! \frac{k \mu(k)}{\varphi(k)} \sum_{\substack{p_{1}<\ldots<p_{i} \\
p_{1} \ldots p_{i} \leq M / k \\
\left(p_{1} \ldots p_{i}, k\right)=1}} \frac{\left(\log p_{1}\right) \ldots\left(\log p_{i}\right)}{p_{1} \ldots p_{i}}\left(\log \widehat{q} k p_{1} \ldots p_{i}\right) \\
& +O\left((\log q)^{i} \frac{k}{\phi(k)}\right) \\
= & (-2)^{i} i ! \frac{k \mu(k)}{\varphi(k)} \sum_{\substack{p_{1}<\ldots<p_{i} \\
p_{1} \ldots p_{i} \leq M / k}} \frac{\left(\log p_{1}\right) \ldots\left(\log p_{i}\right)}{p_{1} \ldots p_{i}}\left(\log \widehat{q} k p_{1} \ldots p_{i}\right) \\
& +O\left((\log q)^{i}\left(\log _{2} q\right) \frac{k}{\phi(k)}\right) \\
= & (-2)^{i} \frac{k \mu(k)}{\varphi(k)} \sum_{\substack{p_{1}, \ldots, p_{i} \\
p_{1} \ldots p_{i} \leq M / k}} \frac{\left(\log p_{1}\right) \ldots\left(\log p_{i}\right)}{p_{1} \ldots p_{i}}\left(\log \widehat{q} k p_{1} \ldots p_{i}\right) \\
& +O\left((\log q)^{i}\left(\log _{2} q\right) \frac{k}{\phi(k)}\right)
\end{aligned}
$$

the error term arising from neglecting the smaller contribution from the primes dividing $k$ and replacing $\varphi(p)^{-1}$ by $p^{-1}$ using the fact that

$$
\sum_{p} \frac{(\log p)^{A}}{p(p-1)}<\infty .
$$

From Mertens's formula, the last sum is equal, up to $O\left((\log q)^{i}\right)$, to the integral

$$
\begin{aligned}
\int_{\substack{y_{1} \geq 0, \ldots, y_{i} \geq 0 \\
y_{1}+\ldots+y_{i} \leq \log (M / k)}} & \left(\log \widehat{q} k+y_{1}+\ldots+y_{i}\right) d y \\
& =(\log \widehat{q} k)\left(\log \frac{M}{k}\right)^{i} \int_{S_{i}} d x+i\left(\log \frac{M}{k}\right)^{i+1} \int_{S_{i}} x_{1} d x .
\end{aligned}
$$

Here $S_{i}=\left\{\left(x_{1}, \ldots, x_{i}\right) \mid x_{j} \geq 0, x_{1}+\ldots+x_{i} \leq 1\right\}$ is the standard $i$-simplex. By induction, one gets immediately

$$
\int_{S_{i}} d x=\frac{1}{i !}, \quad \int_{S_{i}} x_{1} d x=\frac{1}{(i+1) !}
$$


so this contribution to the sum (63) can be written as

$$
\frac{(-2)^{i} \mu(k)}{(i+1) !}\left(\log \frac{M}{k}\right)^{i}\left(\log \widehat{q}^{i+1} M^{i} k\right)+O\left((\log q)^{i}\left(\log _{2} q\right) \frac{k}{\phi(k)}\right)
$$

This is enough to give $y_{k}^{(1)}$; for $y_{k}^{(2)}$ there is an additional sum over primes which, by similar computations, is

$$
\begin{aligned}
-2 \frac{k \mu(k)}{\varphi(k)} \sum_{p \leq M / k} \frac{(\log p)^{2}}{p}(\log \widehat{q} k p)+O\left((\log q)\left(\log _{2} q\right)^{2} \frac{k}{\phi(k)}\right) \\
=-\frac{1}{3} \cdot \frac{k \mu(k)}{\varphi(k)}\left(\log \frac{M}{k}\right)^{2}\left(\log \widehat{q}^{3} M^{2} k\right)+O\left((\log q)^{2} \frac{k}{\phi(k)}\right)
\end{aligned}
$$

and for $y_{k}^{(3)}$ there are two other sums, first

$$
\begin{aligned}
-2 \frac{k \mu(k)}{\varphi(k)} \sum_{p \leq M / k} \frac{(\log p)^{3}}{p}(\log \widehat{q} k p)+O\left((\log q)\left(\log _{2} q\right)^{3} \frac{k}{\phi(k)}\right) \\
=-\frac{1}{6} \cdot \frac{k \mu(k)}{\varphi(k)}\left(\log \frac{M}{k}\right)^{3}\left(\log \widehat{q}^{4} M^{3} k\right)+O\left((\log q)^{3} \frac{k}{\phi(k)}\right)
\end{aligned}
$$

and finally

$$
\begin{aligned}
& 12 \frac{k \mu(k)}{\varphi(k)} \sum_{\substack{p_{1}<p_{2} \\
p_{1} p_{2} \leq M / k}} \frac{\left(\log p_{1} p_{2}\right)\left(\log p_{1}\right)\left(\log p_{2}\right)}{p_{1} p_{2}}\left(\log \widehat{q} k p_{1} p_{2}\right) \\
&+ O\left((\log q)^{2}\left(\log _{2} q\right)^{2} \frac{k}{\phi(k)}\right) \\
&= 12 \frac{k \mu(k)}{\varphi(k)} \sum_{p_{1} p_{2} \leq M / k} \frac{\left(\log p_{1}\right)^{2}\left(\log p_{2}\right)}{p_{1} p_{2}}\left(\log \widehat{q} k p_{1} p_{2}\right) \\
&+O\left((\log q)^{2}\left(\log _{2} q\right)^{2} \frac{k}{\phi(k)}\right) \\
&= \frac{1}{2} \cdot \frac{k \mu(k)}{\varphi(k)}\left(\log \frac{M}{k}\right)^{3}\left(\log \widehat{q}^{4} M^{3} k\right)+O\left((\log q)^{3} \frac{k}{\phi(k)}\right) .
\end{aligned}
$$

From all this we conclude:

Lemma 11. For $i=1,2,3$, we have

$$
y_{k}^{(i)}=c_{i} \frac{k \mu(k)}{\varphi(k)}\left(\log \frac{M}{k}\right)^{i}\left(\log \widehat{q}^{i+1} M^{i} k\right)+O\left((\log q)^{i}\left(\log _{2} q\right) \frac{k}{\phi(k)}\right)
$$

with

$$
c_{1}=-1, \quad c_{2}=1 / 3, \quad c_{3}=0 .
$$


It is now easy to finish the computation of the quadratic form $m_{21}$ for our choice of $y_{k}$.

Lemma 12. With notations as in Lemma 10

$$
\begin{aligned}
\Pi(2,1,0)= & -(\log q)^{6} \Delta^{2}\left(\left(\frac{1}{2}+\Delta\right)^{2}-\Delta\left(\frac{1}{2}+\Delta\right)+\frac{\Delta^{2}}{4}\right) \\
& +O\left((\log q)^{5} \log _{2} q\right), \\
\Pi(1,1,1)= & (\log q)^{6} \Delta^{3}\left(\frac{4}{3}\left(\frac{1}{2}+\Delta\right)^{2}-\Delta\left(\frac{1}{2}+\Delta\right)+\frac{\Delta^{2}}{5}\right) \\
& +O\left((\log q)^{5} \log _{2} q\right), \\
\Pi(1,2,0)= & \frac{1}{3}(\log q)^{6} \Delta^{3}\left(\left(\frac{1}{2}+\Delta\right)^{2}-\Delta\left(\frac{1}{2}+\Delta\right)+\frac{\Delta^{2}}{5}\right) \\
& +O\left((\log q)^{5} \log _{2} q\right), \\
\Pi(0,1,2)= & -\frac{1}{3}(\log q)^{6} \Delta^{4}\left(\frac{3}{2}\left(\frac{1}{2}+\Delta\right)^{2}-\Delta\left(\frac{1}{2}+\Delta\right)+\frac{\Delta^{2}}{6}\right) \\
& +O\left((\log q)^{5} \log _{2}^{3} q\right), \\
\Pi(0,0,3)= & O\left((\log q)^{5} \log _{2}^{3} q\right) .
\end{aligned}
$$

Pr o of. All are similar, so take for instance $\Pi(0,1,2)$; from the previous lemma

$$
\begin{aligned}
\Pi(0,1,2)= & -\frac{1}{3} \sum_{k \leq M} \frac{\mu(k)^{2}}{\varphi(k)}\left(\log \frac{M}{k}\right)^{3}\left(\log \widehat{q}^{3} M^{2} k\right)\left(\log \widehat{q}^{2} M k\right) \\
& +O\left((\log q)^{5}\left(\log _{2} q\right)^{3}\right)
\end{aligned}
$$

and the sum, by summation by parts again, is - up to $O\left((\log q)^{5}\right)$ - the same as the integral

$$
\begin{aligned}
\int_{1}^{M}\left(\log \frac{M}{x}\right)^{3}\left(\log \widehat{q}^{3} M^{2} x\right)\left(\log \widehat{q}^{2} M x\right) \frac{d x}{x} & \\
& =\int_{0}^{\log M} y^{3}(3 \log \widehat{q} M-y)(2 \log \widehat{q} M-y) d y
\end{aligned}
$$

from which the result follows, since moreover $\log \widehat{q}=\log \sqrt{q}+O(1)$.

2.5.3. Diagonalization of $M_{22}$. Recall that

$$
M_{22}=\sum_{b} \frac{1}{b} \sum_{m_{1}, m_{2}} \frac{T\left(m_{1} m_{2}\right)}{m_{1} m_{2}} x_{b m_{1}} x_{b m_{2}}\left(\log \frac{\widehat{Q}}{m_{1} m_{2}}\right) .
$$

Using the multiplicative property of $T$ (see Lemma 5 ), and the fact that $\left(x_{m}\right)$ is supported on squarefree integers, we compute 


$$
\begin{aligned}
& M_{22}=\sum_{b} \frac{1}{b} \sum_{a} \frac{\tau\left(a^{2}\right)}{a^{2}} \sum_{\left(m_{1}, m_{2}\right)=1} \frac{T\left(m_{1} m_{2}\right)}{m_{1} m_{2}} x_{a b m_{1}} x_{a b m_{2}}\left(\log \frac{\widehat{Q}}{a^{2} m_{1} m_{2}}\right) \\
& +\sum_{b} \frac{1}{b} \sum_{a} \frac{T\left(a^{2}\right)}{a^{2}} \sum_{\left(m_{1}, m_{2}\right)=1} \frac{\tau\left(m_{1}\right) \tau\left(m_{2}\right)}{m_{1} m_{2}} x_{a b m_{1}} x_{a b m_{2}}\left(\log \frac{\widehat{Q}}{a^{2} m_{1} m_{2}}\right) \\
& =2 \sum_{b} \frac{1}{b} \sum_{a} \frac{\tau\left(a^{2}\right)}{a^{2}} \sum_{\left(m_{1}, m_{2}\right)=1} \frac{\tau\left(m_{1}\right) T\left(m_{2}\right)}{m_{1} m_{2}} x_{a b m_{1}} x_{a b m_{2}}\left(\log \frac{\widehat{Q}}{a^{2} m_{1} m_{2}}\right) \\
& +\sum_{b} \frac{1}{b} \sum_{a} \frac{T\left(a^{2}\right)}{a^{2}} \sum_{\left(m_{1}, m_{2}\right)=1} \frac{\tau\left(m_{1}\right) \tau\left(m_{2}\right)}{m_{1} m_{2}} x_{a b m_{1}} x_{a b m_{2}}\left(\log \frac{\widehat{Q}}{a^{2} m_{1} m_{2}}\right) \\
& =2 \sum_{b} \frac{1}{b} \sum_{a} \frac{\tau\left(a^{2}\right)}{a^{2}} \sum_{\delta} \frac{\mu(\delta) \tau(\delta)^{2}}{\delta^{2}} \\
& \times \sum_{m_{1}, m_{2}} \frac{\tau\left(m_{1}\right) T\left(m_{2}\right)}{m_{1} m_{2}} x_{a b \delta m_{1}} x_{a b \delta m_{2}}\left(\log \frac{\widehat{Q}}{a^{2} \delta^{2} m_{1} m_{2}}\right) \\
& +2 \sum_{b} \frac{1}{b} \sum_{a} \frac{\tau\left(a^{2}\right)}{a^{2}} \sum_{\delta} \frac{\mu(\delta) \tau(\delta) T(\delta)}{\delta^{2}} \\
& \times \sum_{m_{1}, m_{2}} \frac{\tau\left(m_{1}\right) \tau\left(m_{2}\right)}{m_{1} m_{2}} x_{a b \delta m_{1}} x_{a b \delta m_{2}}\left(\log \frac{\widehat{Q}}{a^{2} \delta^{2} m_{1} m_{2}}\right) \\
& +\sum_{b} \frac{1}{b} \sum_{a} \frac{T\left(a^{2}\right)}{a^{2}} \sum_{\delta} \frac{\mu(\delta) \tau(\delta)^{2}}{\delta^{2}} \\
& \times \sum_{m_{1}, m_{2}} \frac{\tau\left(m_{1}\right) \tau\left(m_{2}\right)}{m_{1} m_{2}} x_{a b \delta m_{1}} x_{a b \delta m_{2}}\left(\log \frac{\widehat{Q}}{a^{2} \delta^{2} m_{1} m_{2}}\right) .
\end{aligned}
$$

Let $m_{22}$ denote the part of the first term arising by using

$$
\log \frac{\widehat{Q}}{a^{2} \delta^{2} m_{1} m_{2}}=\log \frac{\widehat{Q}}{m_{1} m_{2}}-2 \log a \delta ;
$$

this will be the main contribution: all the other terms can be directly estimated and shown to be of order of magnitude at most $(\log q)^{5} \log _{2} q$.

We have

$$
m_{22}=2 \sum_{k} \nu(k) \sum_{m_{1}, m_{2}} \frac{\tau\left(m_{1}\right) T\left(m_{2}\right)}{m_{1} m_{2}} x_{k m_{1}} x_{k m_{2}}\left(\log \frac{\widehat{Q}}{m_{1} m_{2}}\right)
$$

since, for squarefree $k$,

$$
\frac{1}{k} \sum_{a b \delta=k} \frac{\mu(\delta) \tau(\delta)^{2} \tau\left(a^{2}\right)}{a \delta}=\frac{1}{k} \prod_{p}\left(1-\frac{4}{p}+\frac{3}{p}\right)=\nu(k) .
$$


The treatment is now similar to that of $m_{21}$ : define

$$
z_{k}:=z_{k}^{(0)}:=\sum_{m} \frac{T(m)}{m} x_{k m}, \quad z_{k}^{(1)}:=\sum_{m} \frac{T(m)}{m}(\log m) x_{k m}
$$

and

$$
\widetilde{\Pi}(a, b, c)=(\log \widehat{Q})^{a} \sum_{k} \nu(k) y_{k}^{(b)} z_{k}^{(c)}
$$

then

$$
m_{22}=2(\widetilde{\Pi}(1,0,0)-\widetilde{\Pi}(0,1,0)-\widetilde{\Pi}(0,0,1)) .
$$

Lemma 13. We have

$$
\begin{aligned}
z_{k} & =2 \sum_{l \leq M / k} \frac{(\log l) \Lambda(l)}{l} y_{k l}, \\
z_{k}^{(1)} & =\sum_{l \leq M / k} \frac{\tau(l) \Lambda(l)}{l} z_{k l}+\sum_{l \leq M / k} \frac{T(l) \Lambda(l)}{l} y_{k l} .
\end{aligned}
$$

Proof. For the first one, (60) implies

$$
z_{k}=\sum_{l}\left(\sum_{m n=l} \frac{T(m)}{m} g(n)\right) y_{k l}
$$

and the Dirichlet generating series for the coefficient of $l$ is $L(s+1)$ where

$$
L(s)=\zeta(s)^{-2} \sum_{n} T(n) n^{-s} .
$$

From the first part of Lemma 5, we get

$$
\sum_{n} T(n) n^{-s}=4 \zeta \zeta^{\prime \prime}-2\left(\zeta \zeta^{\prime}\right)^{\prime}=2\left(\zeta \zeta^{\prime \prime}-\left(\zeta^{\prime}\right)^{2}\right)
$$

so

$$
L(s)=2\left(\zeta^{\prime} \zeta^{-1}\right)^{\prime} .
$$

As to $z_{k}^{(1)}$, write $\log m=\sum_{a b=m} \Lambda(a)$ and use again the multiplicative property of $T$.

2.5.4. Evaluation of $m_{22}$. The mollifier was defined by (62).

LEMMA 14. We have

$$
\begin{aligned}
z_{k} & =-\frac{1}{3} \cdot \frac{k \mu(k)}{\varphi(k)}\left(\log \frac{M}{k}\right)^{2}\left(\log \widehat{q}^{3} M^{2} k\right)+O\left((\log q)^{2} \frac{k}{\phi(k)}\right) \\
& =-y_{k}^{(2)}+O\left(\frac{k}{\varphi(k)}(\log q)^{2} \log _{2} q\right)
\end{aligned}
$$

and

$$
z_{k}^{(1)}=O\left(\frac{k}{\varphi(k)}(\log q)^{3}\right)
$$


Proof. We will be brief: on the one hand

$$
\begin{aligned}
z_{k} & =-2 \frac{k \mu(k)}{\varphi(k)} \sum_{p \leq M / k} \frac{(\log p)^{2}}{p} \log \widehat{q} k p+O\left(\frac{k}{\varphi(k)}\left(\log _{2} q\right)^{3}\right) \\
& =-2 \frac{k \mu(k)}{\varphi(k)} \int_{0}^{\log (M / k)} y(y+\log \widehat{q} k) d y+O\left(\frac{k}{\varphi(k)}(\log q)^{2}\right) \\
& =-\frac{1}{3} \cdot \frac{k \mu(k)}{\varphi(k)}\left(\log \frac{M}{k}\right)^{2}\left(\log \widehat{q}^{3} M^{2} k\right)+O\left(\frac{k}{\varphi(k)}(\log q)^{2}\right)
\end{aligned}
$$

and on the other hand the two contributions to $z_{k}^{(1)}$ are respectively (using the previous computation)

$$
\begin{aligned}
\frac{1}{3} \cdot \frac{k \mu(k)}{\varphi(k)} \sum_{p \leq M / k} & \frac{2 \log p}{p}\left(\log \frac{M}{p}\right)^{2}\left(\log \widehat{q}^{3} M^{2} p\right) \\
= & \frac{1}{6} \cdot \frac{k \mu(k)}{\varphi(k)}\left(\log \frac{M}{k}\right)^{3}\left(\log \widehat{q}^{4} M^{3} k\right)+O\left(\frac{k}{\varphi(k)}(\log q)^{3}\right)
\end{aligned}
$$

and (this is the same as one of the sums considered in $y_{k}^{(3)}$ )

$$
\begin{aligned}
-\frac{k \mu(k)}{\varphi(k)} & \sum_{p \leq M / k} \frac{2(\log p)^{3}}{p}(\log \widehat{q} k p) \\
& =-\frac{1}{6} \cdot \frac{k \mu(k)}{\varphi(k)}\left(\log \frac{M}{k}\right)^{3}\left(\log \widehat{q}^{4} M^{3} k\right)+O\left(\frac{k}{\varphi(k)}(\log q)^{3}\right) .
\end{aligned}
$$

From this (referring to Lemma 12), we obtain

$$
\begin{aligned}
\widetilde{\Pi}(1,0,0) & =-(\log \widehat{Q}) \sum_{k} \nu(k) y_{k} y_{k}^{(2)}+O\left((\log q)^{5}\right) \\
& =-\Pi(1,2,0)+O\left((\log q)^{5}\right), \\
\widetilde{\Pi}(0,1,0) & =-\sum_{k} \nu(k) y_{k}^{(1)} y_{k}^{(2)}+O\left((\log q)^{5}\right) \\
& =-\Pi(0,1,2)+O\left((\log q)^{5}\right), \\
\widetilde{\Pi}(0,0,1) & =O\left((\log q)^{5}\right) .
\end{aligned}
$$

2.5.5. The case of $N_{2}$. For $N_{2}$ and $N_{1}$, the situation is much simpler. Recall the decomposition (58). We have

$$
\Pi(1,0,0)=\sum_{k} \nu(k) y_{k}^{2}
$$


where $y_{k}$ is as before, and

$$
N_{1}=\sum_{k} j(k) y_{k}
$$

with $j(k)=\mu(k) / k$. Hence we select

$$
y_{k}=\frac{j(k)}{\nu(k)}=\frac{k \mu(k)}{\varphi(k)}
$$

for $k \leq M$, to optimize $\Pi(1,0,0)$ with respect to $N_{1}$. We then have $\Pi(1,0,0)=(\log M)(\log \widehat{Q})+O(1), \quad N_{1}=\log M+O(1)=\Delta \log q+O(1)$. Moreover $\Pi(0,1,0)=\sum \nu(k) y_{k} y_{k}^{(1)}$ and proceeding as before we evaluate $y_{k}^{(1)}$, namely

$$
y_{k}^{(1)}=-2 \frac{k \mu(k)}{\varphi(k)}\left(\log \frac{M}{k}\right)+O\left(\frac{k}{\varphi(k)} \log _{2} q\right) .
$$

Finally we find $\Pi(0,1,0)=2(\log M)^{2}+O\left((\log q) \log _{2} q\right)$ by summation by parts, and

Hence

$$
N_{2}=\Delta(1+2 \Delta)(\log q)^{2}+O\left((\log q) \log _{2} q\right)
$$

$$
\frac{N_{1}^{2}}{N_{2}}=\frac{\Delta}{1+2 \Delta}+O\left(\frac{\log _{2} q}{\log q}\right)
$$

Letting $\Delta \rightarrow 1 / 4$ we obtain the harmonic analogue of Theorem 2 .

2.6. Conclusion. To summarize our computations, consider the two polynomials in the variable $\Delta$ :

$$
\begin{aligned}
M_{1}(\Delta):= & \Delta\left(\frac{\Delta^{2}}{3}+\frac{\Delta}{2}+\frac{1}{4}\right), \\
M_{2}(\Delta):= & \frac{1}{12} M_{1}(\Delta)+\frac{1}{2} \Delta^{2}\left(\left(\frac{1}{2}+\Delta\right)^{2}-\Delta\left(\frac{1}{2}+\Delta\right)+\frac{\Delta^{2}}{4}\right) \\
& +\frac{1}{2} \Delta^{3}\left(\frac{4}{3}\left(\frac{1}{2}+\Delta\right)^{2}-\Delta\left(\frac{1}{2}+\Delta\right)+\frac{\Delta^{2}}{5}\right) \\
& +\frac{1}{3} \Delta^{3}\left(\left(\frac{1}{2}+\Delta\right)^{2}-\Delta\left(\frac{1}{2}+\Delta\right)+\frac{\Delta^{2}}{5}\right) \\
& +\frac{1}{3} \Delta^{4}\left(\frac{3}{2}\left(\frac{1}{2}+\Delta\right)^{2}-\Delta\left(\frac{1}{2}+\Delta\right)+\frac{\Delta^{2}}{6}\right) \\
= & \frac{2}{9} \Delta^{6}+\frac{2}{3} \Delta^{5}+\frac{5}{6} \Delta^{4}+\frac{19}{36} \Delta^{3}+\frac{1}{6} \Delta^{2}+\frac{1}{48} \Delta
\end{aligned}
$$

Then it follows from Lemma 10 that for $\Delta<1 / 2$,

$$
M_{1}=M_{1}(\Delta)(\log q)^{3}+O\left((\log q)^{2}\right)
$$


and from (45), (51), (47), (57), Lemma 12, (67)-(69) (and the computations to check (49) and (55), which have to be done but are simple consequences of the previous estimations $\left({ }^{4}\right)$ ) that for $\Delta<1 / 4$, we have

$$
M_{2}=M_{2}(\Delta)(\log q)^{6}+O\left((\log q)^{5} \log _{2} q\right) .
$$

Now, partial fraction decomposition yields

$$
\frac{M_{1}(\Delta)^{2}}{M_{2}(\Delta)}=\frac{1}{2}\left(1-\frac{1}{(1+2 \Delta)^{3}}\right) \text {. }
$$

Hence the harmonic analogue of Theorem 3 follows, in the more precise form

$$
\sum_{L(f, 1 / 2)=0, L^{\prime}(f, 1 / 2) \neq 0}^{\mathrm{h}} 1 \geq \frac{1}{2}\left(1-\frac{1}{(1+2 \Delta)^{3}}\right)+O\left(\frac{\log _{2} q}{\log q}\right) .
$$

3. Non-vanishing in natural average. We now consider the first and second moments for the natural average

$$
M_{1}^{\mathrm{n}}:=\sum_{L(f, 1 / 2)=0} M(f) L^{\prime}(f, 1 / 2), \quad M_{2}^{\mathrm{n}}:=\sum_{L(f, 1 / 2)=0}\left|M(f) L^{\prime}(f, 1 / 2)\right|^{2} .
$$

To get from the harmonic averages to the natural average, we use the method of [KM1]. For $x \geq 1$, let

$$
\omega_{f}(x):=\sum_{d l^{2} \leq x} \frac{\lambda_{f}\left(d^{2}\right)}{d l^{2}}
$$

(a partial sum of the value of the symmetric square $L$-function of $f$ at $s=1$ ). Applying Proposition 2 of [KM1], it follows that for $x=q^{\varepsilon}, \varepsilon>0$ being small enough, we have

$$
\begin{aligned}
M_{1}^{\mathrm{n}} & =\frac{q}{2 \pi^{2}} \sum_{L(f, 1 / 2)=0}^{\mathrm{h}} \omega_{f}(x) M(f) L^{\prime}(f, 1 / 2)+O\left(q^{-c}\right), \\
M_{2}^{\mathrm{n}} & =\frac{q}{2 \pi^{2}} \sum_{L(f, 1 / 2)=0}^{\mathrm{h}} \omega_{f}(x)\left|M(f) L^{\prime}(f, 1 / 2)\right|^{2}+O\left(q^{-c}\right),
\end{aligned}
$$

for some $c=c(\varepsilon)$.

To check the conditions of [KM1, Prop. 2], we use the growth condition

$$
x_{m} \ll(\tau(m) \log q m)^{A}
$$

together with the estimates

$$
\begin{aligned}
\frac{1}{(f, f)} & \ll(\log q) q^{-1}, \\
L^{\prime}(f, 1 / 2) & \ll q^{1 / 4}(\log q)^{2}
\end{aligned}
$$

$\left({ }^{4}\right)$ Count the number of logarithms and estimate directly. 
to obtain the individual bounds, and with Propositions 2 and 6 for the average bounds (see [Kow, Lemma 43] for more details).

We write

$$
\begin{aligned}
& \widetilde{M}_{1}=\sum_{L(f, 1 / 2)=0}^{\mathrm{h}} \omega_{f}(x) M(f) L^{\prime}(f, 1 / 2), \\
& \widetilde{M}_{2}=\sum_{L(f, 1 / 2)=0}^{\mathrm{h}} \omega_{f}(x)\left|M(f) L^{\prime}(f, 1 / 2)\right|^{2} .
\end{aligned}
$$

3.1. Computing the first moment. Using the formula

$$
\lambda_{f}(m) \lambda_{f}\left(d^{2}\right)=\sum_{r \mid\left(m, d^{2}\right)} \varepsilon_{q}(r) \lambda_{f}\left(\frac{m d^{2}}{r^{2}}\right)
$$

we get, for $M=q^{\Delta}$ with $\Delta<1 / 2$,

$$
\widetilde{M}_{1}=\sum_{m \leq M} \frac{x_{m}}{m} \sum_{d l^{2} \leq x} \frac{1}{d^{2} l^{2}} \sum_{r \mid\left(d^{2}, m\right)} r \log \left(\widehat{q} r^{2} /\left(d^{2} m\right)\right)+O\left(q^{-c}\right) .
$$

We remove the constraint $d l^{2} \leq x$ at the cost of an admissible error term (that is, $O\left(q^{-c}\right)$ ). Since $m$ is squarefree, $r\left|d^{2} \Leftrightarrow r\right| d$ and we get, setting $d^{\prime}=d / r$

$$
\text { (73) } \begin{aligned}
\widetilde{M}_{1}= & \zeta(2) \sum_{m \leq M} \frac{x_{m} d_{-1}(m)}{m} \sum_{d^{\prime}} \frac{1}{d^{\prime 2}}\left(\log \widehat{q} m-2 \log d^{\prime}\right)+O\left(q^{-c}\right) \\
= & \zeta(2)^{2} \sum_{m \leq M} \frac{x_{m} d_{-1}(m)}{m} \log \widehat{q} m+2 \zeta(2) \zeta^{\prime}(2) \sum_{m \leq M} \frac{x_{m} d_{-1}(m)}{m} \\
& +O\left(q^{-c}\right) .
\end{aligned}
$$

3.2. Computing $\widetilde{M}_{2}$. We have

$$
\widetilde{M}_{2}=\sum_{b} \frac{1}{b} \sum_{m_{1}, m_{2} \leq M} \frac{x_{b m_{1}} x_{b m_{2}}}{\sqrt{m_{1} m_{2}}} \sum_{d l^{2} \leq x} \sum_{r \mid\left(d^{2}, m_{1} m_{2}\right)} X\left(\frac{d^{2} m_{1} m_{2}}{r^{2}}\right) .
$$

By Proposition 5, the second moment decomposes in a way similar to (45): for $M=q^{\Delta}, \Delta<1 / 4$,

$$
\widetilde{M}_{2}=\frac{1}{12} \widetilde{M}_{21}-\frac{1}{4} \widetilde{M}_{22}+\widetilde{M}_{3}+O\left(q^{-c}\right)
$$

where

$$
\begin{aligned}
\widetilde{M}_{21}=\sum_{b} \frac{1}{b} \sum_{d l^{2} \leq x} \frac{1}{d^{2} l^{2}} \sum_{m_{1}, m_{2} \leq M} \sum_{r \mid\left(d^{2}, m_{1} m_{2}\right)} r \frac{\tau\left(m_{1} m_{2} d^{2} / r^{2}\right)}{m_{1} m_{2}} \\
\times x_{b m_{1}} x_{b m_{2}}\left(\log \frac{\widehat{Q} r^{2}}{m_{1} m_{2} d^{2}}\right)^{3},
\end{aligned}
$$


(76)

$$
\begin{aligned}
\widetilde{M}_{22}=\sum_{b} \frac{1}{b} \sum_{d l^{2} \leq x} \frac{1}{d^{2} l^{2}} \sum_{m_{1}, m_{2}} \sum_{r \mid\left(d^{2}, m_{1} m_{2}\right)} r \frac{T\left(m_{1} m_{2} d^{2} / r^{2}\right)}{m_{1} m_{2}} \\
\times x_{b m_{1}} x_{b m_{2}}\left(\log \frac{\widehat{Q} r^{2}}{m_{1} m_{2} d^{2}}\right), \\
\widetilde{M}_{3}=\sum_{b} \frac{1}{b} \sum_{d l^{2} \leq x} \frac{1}{d^{2} l^{2}} \sum_{m_{1}, m_{2}} \sum_{r \mid\left(d^{2}, m_{1} m_{2}\right)} r \frac{\tau\left(m_{1} m_{2} d^{2} / r^{2}\right)}{m_{1} m_{2}} \\
\times P_{1}\left(\log \frac{\widehat{Q} r^{2}}{m_{1} m_{2} d^{2}}\right)
\end{aligned}
$$

(compare with (47), (48)). As in [KM1, 4.5], we drop the constraint $d l^{2} \leq x$ in $(75)-(77)$ at the cost of an error term which is $O\left(q^{-c}\right)$.

As before, the strategy is now to optimize the quadratic form $\widetilde{M}_{21}$ with respect to the linear form (73). For this, we shall need properties of some "quasi-multiplicative" arithmetic functions. For a more detailed treatment, see [Kow, 6.2].

3.2.1. Some quasi-multiplicative functions. During the transformation process of $\widetilde{M}_{21}$ we will meet expressions of the following type, where $r$ stands for a divisor of $m_{1} m_{2}$ :

with

$$
\sum_{\substack{d \geq 1 \\ d^{2} \equiv 0 \bmod r}} \frac{\tau\left(d^{2} / r\right)}{d^{2}}\left(\log d^{2}\right)^{k}=(-1)^{k}(g(\delta, r))_{\delta=0}^{(k)}
$$

$k$ is an integer).

$$
g(\delta, r):=\sum_{\substack{d \geq 1 \\ d^{2} \equiv 0 \bmod r}} \frac{\tau\left(d^{2} / r\right)}{d^{2+2 \delta}}
$$

This Dirichlet series is computed in [KM1, Lemma 13] (it is the case $\delta=0$ ), and is the product of a constant and a multiplicative function $\kappa(\delta, r)$ :

$$
g(\delta, r)=\frac{\zeta(2+2 \delta)^{3}}{\zeta(4+4 \delta)} \kappa(\delta, r), \quad \kappa(\delta, r)=\prod_{p \mid r} \frac{1}{p^{2+2 \delta}} \prod_{p \| r} \frac{2}{1+p^{-2(1+\delta)}} .
$$

For an integer $k \geq 0$, let $\kappa^{(k)}(r):=(\kappa(\delta, r))_{\delta=0}^{(k)}$ be the $k$ th derivative of $\delta \mapsto \kappa(\delta, r)$ at $\delta=0$. By Leibniz's rule, the series $(g(\delta, r))_{\delta=0}^{(k)}$ is a linear combination of the $\kappa^{\left(k^{\prime}\right)}(r)$, for $k^{\prime} \leq k$.

In the next steps, we shall use the following two properties of $\kappa^{(k)}(r)$ : the bound

$$
\kappa^{(k)}(r) \ll_{k} \frac{\tau(r)(\log r)^{k}}{\prod_{p \mid r} p^{2}}
$$


and the quasi-multiplicativity

$$
\kappa^{(k)}\left(r_{1} r_{2}\right)=\sum_{k^{\prime}+k^{\prime \prime}=l}\left(\begin{array}{c}
k \\
k^{\prime}
\end{array}\right) \kappa^{\left(k^{\prime}\right)}\left(r_{1}\right) \kappa^{\left(k^{\prime \prime}\right)}\left(r_{2}\right) \quad \text { for }\left(r_{1}, r_{2}\right)=1 .
$$

We will also encounter the arithmetic convolution $f^{(j)}=\mu *\left(\operatorname{Id} \times \log ^{j}\right)$. This last function also enjoys quasi-multiplicative properties:

$$
f^{(j)}\left(r_{1} r_{2}\right)=\sum_{j^{\prime}+j^{\prime \prime}=j}\left(\begin{array}{l}
j \\
j^{\prime}
\end{array}\right) f^{\left(j^{\prime}\right)}\left(r_{1}\right) f^{\left(j^{\prime \prime}\right)}\left(r_{2}\right) \quad \text { for }\left(r_{1}, r_{2}\right)=1 .
$$

The following formula will be used to separate $m_{1} m_{2} / r$ and $d^{2} / r$.

$$
\sum_{r \mid(m, n)} r(\log r)^{j} \tau\left(\frac{m n}{r^{2}}\right)=\sum_{r \mid(m, n)} f^{(j)}(r) \tau\left(\frac{m}{r}\right) \tau\left(\frac{n}{r}\right) .
$$

3.3. The preferred quadratic form II. Decompose $\log \left(\widehat{Q} r^{2} /\left(m_{1} m_{2} d^{2}\right)\right)=$ $\log \left(\widehat{Q} /\left(m_{1} m_{2}\right)\right)+\log r^{2}-\log d^{2}:=U+V+W$, say. We have $(U+V+W)^{3}=$ $\sum_{i+j+k=3} c_{i, j, k} U^{i} V^{j} W^{k}$, and $\widetilde{M}_{21}$ decomposes accordingly:

$$
\widetilde{M}_{21}=\sum_{i+j+k=3} c_{i, j, k} \widetilde{M}_{21}^{i, j, k}
$$

3.3.1. Decomposing $\widetilde{M}_{21}$. Using the results of the preceding section and (80), we decompose $\widetilde{M}_{21}$ into pieces of the form

$$
\sum_{b} \frac{1}{b} \sum_{m_{1}, m_{2}} \frac{x_{b m_{1}} x_{b m_{2}}}{m_{1} m_{2}}\left(\log \frac{\widehat{Q}}{m_{1} m_{2}}\right)^{i} \sum_{r \mid m_{1} m_{2}} f^{(j)} \kappa^{(k)}(r) \tau\left(\frac{m_{1} m_{2}}{r}\right)
$$

where $i, j, k$ are integers satisfying $0 \leq i, j, k, i+j+k \leq 3$.

Note at this point (use the quasi-multiplicativity of $f^{(j)}, \kappa^{(k)}$, and the multiplicativity of $\tau$ ) that the convolution $\tau * f^{(j)} \kappa^{(k)}$ is quasi-multiplicative, in the following sense: for $(m, n)=1, \tau * f^{(j)} \kappa^{(k)}(m n)$ is a linear combination (with at most $j k$ terms) of products $\tau * f^{\left(j^{\prime}\right)} \kappa^{\left(k^{\prime}\right)}(m) \times \tau * f^{\left(j^{\prime \prime}\right)} \kappa^{\left(k^{\prime \prime}\right)}(n)$ with $j^{\prime}+j^{\prime \prime} \leq j$ and $k^{\prime}+k^{\prime \prime} \leq k$.

Then $m_{1}$ and $m_{2}$ decompose uniquely into $m_{1}=m_{1}^{\prime} m_{3}, m_{2}=m_{2}^{\prime} m_{3}$ with $\left(m_{1}^{\prime}, m_{2}^{\prime}\right)=1$, and we further have $\left(m_{1}^{\prime}, m_{2}^{\prime}, m_{3}\right)=1$ since $m_{1}, m_{2}$ were assumed to be squarefree. Define $h^{j, k}$ by

$$
h^{j, k}(m)=\frac{1}{m} \tau * f^{(j)} \kappa^{(k)}(m) ;
$$

then we see by quasi-multiplicativity that $\widetilde{M}_{21}$ is a linear combination of the quadratic forms 


$$
\begin{aligned}
\sum_{b} \frac{1}{b} \sum_{\substack{m_{1}, m_{2}, m_{3} \\
\left(m_{1}, m_{2}\right)=1}} x_{b m_{3} m_{1}} x_{b m_{3} m_{2}} h^{j_{1}, k_{1}}\left(m_{1}\right) h^{j_{2}, k_{2}}\left(m_{2}\right) h^{j_{3}, k_{3}}\left(m_{3}^{2}\right) \\
\times\left(\log \frac{\widehat{Q}}{m_{1} m_{2} m_{3}^{2}}\right)^{i},
\end{aligned}
$$

with $i+j_{1}+k_{1}+j_{2}+k_{2}+j_{3}+k_{3} \leq 3$.

Finally, we detect the remaining condition $\left(m_{1}, m_{2}\right)=1$ using the Möbius function, and obtain, after expanding the factor $\left(\log \left(\widehat{Q} /\left(m_{1} m_{2} m_{3}^{2}\right)\right)\right)^{i}$, the decomposition of $\widetilde{M}_{21}$ as a linear combination of the quadratic forms

$$
\begin{aligned}
& \widetilde{M}_{21}^{i, \mathbf{i}, \mathbf{j}, \mathbf{k}} \\
& \quad:=\sum_{k} \nu^{\mathbf{i}, \mathbf{j}, \mathbf{k}}(k) \sum_{m_{1}, m_{2}} x_{k m_{1}} x_{k m_{2}} h^{j_{4}, k_{4}}\left(m_{1}\right) h^{j_{5}, k_{5}}\left(m_{2}\right)\left(\log \frac{\widehat{Q}}{m_{1} m_{2}}\right)^{i}
\end{aligned}
$$

with

$$
\begin{gathered}
\mathbf{i}=\left(i_{1}, i_{3}\right), \quad \mathbf{j}=\left(j_{1}, j_{2}, j_{3}, j_{4}, j_{5}\right), \quad \mathbf{k}=\left(k_{1}, k_{2}, k_{3}, k_{4}, k_{5}\right), \\
i+i_{1}+i_{3}+j_{1}+j_{2}+j_{3}+j_{4}+j_{5}+k_{1}+k_{2}+k_{3}+k_{4}+k_{5} \leq 3
\end{gathered}
$$

and

$$
i, i_{1}, \ldots, k_{5} \geq 0
$$

More precisely, we have

$$
\widetilde{M}_{21}=\frac{\zeta(2)^{4}}{\zeta(4)} \widetilde{M}_{21}^{3,(0,0),(0,0,0),(0,0,0)}+\sum_{i<3} \sum_{\mathbf{i}, \mathbf{j}, \mathbf{k}} c_{i, \mathbf{i}, \mathbf{j}, \mathbf{k}} \widetilde{M}_{21}^{i, \mathbf{i}, \mathbf{j}, \mathbf{k}} .
$$

To ease the notations, set

$$
\widetilde{M}:=\widetilde{M}_{21}^{3,(0,0),(0,0,0),(0,0,0)} .
$$

For the optimal choice of the vector $\left(x_{m}\right)$, the main contribution to $\widetilde{M}_{21}$ will be seen to come from $\widetilde{M}$ : for $i<3$ we will check that

$$
\widetilde{M}_{21}^{i, \mathbf{i}, \mathbf{j}, \mathbf{k}}=O\left(\widetilde{M} \frac{\log _{2} q}{\log q}\right) .
$$

Let us now concentrate on $\widetilde{M}$.

3.4. Diagonalization of $\widetilde{M}$. Opening the factor $\left(\log \left(\widehat{Q} /\left(m_{1} m_{2}\right)\right)\right)^{3}$, we have the decomposition

$$
\begin{aligned}
\widetilde{M}= & \widetilde{\Pi}(3,0,0)-6 \widetilde{\Pi}(2,1,0)+6 \widetilde{\Pi}(1,1,1)+6 \widetilde{\Pi}(1,2,0) \\
& -6 \widetilde{\Pi}(0,1,2)-2 \widetilde{\Pi}(0,0,3) .
\end{aligned}
$$

3.4.1. Optimizing $\widetilde{\Pi}(3,0,0)$. Set

$$
\widetilde{h}(m)=h^{0,0}(m), \quad \widetilde{\nu}(k):=\nu^{(0,0),(0,0,0),(0,0,0)}(k),
$$


write $\widetilde{g}$ for the convolution inverse of $\widetilde{h}$, and set

$$
\begin{aligned}
\widetilde{\jmath}(k): & =\widetilde{g} * \frac{d_{-1} \log (\widehat{q} / \mathrm{Id})}{\mathrm{Id}}(k)=\sum_{a b=k} \widetilde{g}(a) \log (\widehat{q} / b) \frac{d_{-1}(b)}{b}, \\
j_{0}(k): & =\widetilde{g} * \frac{d_{-1}}{\mathrm{Id}}(k)=\sum_{a b=k} \widetilde{g}(a) \frac{d_{-1}(b)}{b} .
\end{aligned}
$$

After some computations, it is found that for $p$ prime we have

$$
\begin{aligned}
\widetilde{\jmath}(p)=-A\left(p^{-1}\right) & \text { with } A=X \frac{(1-X)(1+X)^{2}}{1+X^{2}}, \\
\widetilde{\nu}(p)=B\left(p^{-1}\right) & \text { with } B=\frac{\left(1-X^{2}\right)^{3}}{\left(1+X^{2}\right)^{2}} .
\end{aligned}
$$

The next lemma is the analogue of Lemma 9:

LEMma 15. For all squarefree integer $k$ we have

$$
\widetilde{\jmath}(k)=j_{0}(k)(\log \widehat{q} k+O(1)) .
$$

Proof. We have

$$
\begin{aligned}
\widetilde{\jmath}(k) & =j_{0}(k) \log \widehat{q}-\sum_{a b=k} g(a) \frac{d_{-1}(b)}{b} \log b \\
& =j_{0}(k) \log \widehat{q}-\sum_{p \mid k} \frac{\log p}{p} d_{-1}(p) j_{0}(k / p) \\
& =j_{0}(k)\left(\log \widehat{q}-\sum_{p \mid k} \frac{(\log p) d_{-1}(p)}{p j_{0}(p)}\right) \\
& =j_{0}(k)\left(\log \widehat{q}+\sum_{p \mid k} \log p \frac{\left(1+1 / p^{2}\right)}{\left(1-1 / p^{2}\right)}\right) .
\end{aligned}
$$

With our notations we have

$$
\widetilde{\Pi}(3,0,0)=(\log \widehat{Q})^{3} \sum_{k} \widetilde{\nu}(k) \sum_{m_{1}, m_{2} \leq M} \widetilde{h}\left(m_{1}\right) \widetilde{h}\left(m_{2}\right) x_{k m_{1}} x_{k m_{2}} .
$$

To diagonalize $\widetilde{\Pi}(3,0,0)$ we make the - now classical - change of variable

$$
y_{k}=\sum_{m} \widetilde{h}(m) x_{k m},
$$

so that

$$
x_{m}=\sum_{k} \widetilde{g}(k) y_{k m} \quad \text { and } \quad \widetilde{\Pi}(3,0,0)=(\log \widehat{Q})^{3} \sum_{k} \widetilde{\nu}(k) y_{k}^{2} .
$$


We now choose $\left(y_{k}\right)$ optimally to optimize $\widetilde{\Pi}(3,0,0)$ with respect to $\widetilde{M}_{1}$ :

$$
y_{k}=\mu(k)^{2} \frac{\widetilde{\jmath}(k)}{\widetilde{\nu}(k)} \quad \text { for } k \leq M .
$$

We immediately see that the corresponding $\left(x_{m}\right)$ satisfies condition (8), and (73) gives

$$
\begin{aligned}
\widetilde{M}_{1} & =\zeta(2)^{2} \sum_{k \leq M} \widetilde{\jmath}(k) y_{k}+2 \zeta(2) \zeta^{\prime}(2) \sum_{k \leq M} j_{0}(k) y_{k} \\
& =\zeta(2)^{2} \sum_{k} \mu(k)^{2} \frac{\widetilde{\jmath}(k)^{2}}{\widetilde{\nu}(k)}+2 \zeta(2) \zeta^{\prime}(2) \sum_{k} \mu(k)^{2} \frac{\widetilde{\jmath}(k) j_{0}(k)}{\widetilde{\nu}(k)} \\
& =\zeta(2)^{2} \sum_{k \leq M} \mu(k)^{2} \frac{j_{0}(k)^{2}}{\widetilde{\nu}(k)}\left(\log ^{2}(\widehat{q} k)+O(\log \widehat{q} k)\right) \\
& =\frac{\zeta(2)^{3}}{\zeta(4)} \Delta\left(\frac{\Delta^{2}}{3}+\frac{\Delta}{2}+\frac{1}{4}\right)(\log q)^{3}+O\left((\log q)^{2}\right) .
\end{aligned}
$$

In the last two lines, we have used Lemma 15, and a partial summation exactly similar to that performed in Lemma 10 with the following variant:

$$
\sum_{k \leq M} \mu(k)^{2} \frac{j_{0}(k)^{2}}{\widetilde{\nu}(k)}=\frac{\zeta(2)}{\zeta(4)} \log M+O(1),
$$

which follows by computing the residue at $s=0$ of the Dirichlet series

$$
\sum_{k \geq 1} \frac{\mu(k)^{2} j_{0}(k)^{2}}{\widetilde{\nu}(k)} k^{-s}=\prod_{p}\left(1+\frac{2 p^{-(s+1)}}{\left(1+p^{-(s+1)}\right)(p-1)}\right)
$$

which has analytic continuation to $\operatorname{Re}(s)>-1$. Similarly, we have

$$
\widetilde{\Pi}(3,0,0)=\frac{\zeta(2)}{\zeta(4)} \Delta\left(\frac{\Delta^{2}}{3}+\frac{\Delta}{2}+\frac{1}{4}\right)(\log q)^{6}+O\left((\log q)^{5}\right) .
$$

3.4.2. Estimation of $\widetilde{\Pi}(u, v, w)$. We set

$$
y_{k}^{(i)}=\sum_{m} \widetilde{h}(m)(\log m)^{i} x_{k m} .
$$

The next lemma is the analogue of Lemma 11, and its proof is exactly the same, using Lemma 15 , and the equality $\widetilde{h}(p)=\tau(p) / p+O\left(p^{-2}\right)$.

Lemma 16. For $i=1,2,3$, we have

$$
y_{k}^{(i)}=c_{i} \frac{j_{0}(k)}{\nu(k)}\left(\log \frac{M}{k}\right)^{i}\left(\log \widehat{q}^{i+1} M^{i} k\right)+O\left(\frac{j_{0}(k)}{\nu(k)}(\log q)^{i} \log _{2} q\right)
$$

with

$$
c_{1}=-1, \quad c_{2}=1 / 3, \quad c_{3}=0 .
$$


Then the computations of Section 2.5.2 hold verbatim and we get at the vector defined by the quadratic form

$$
\widetilde{M}_{21}=\frac{\zeta(2)^{5}}{\zeta(4)^{2}} M_{21}+O\left((\log q)^{5} \log _{2} q\right)
$$

where by $\widetilde{M}_{21}$ we mean the value of the quadratic form at the vector $\left(x_{m}\right)$ in (86) and (87), and similarly for $M_{21}$ and the vector in (60) and (62). Similarly, one can show, with the same abuse of notation, that

$$
\widetilde{M}_{22}=\frac{\zeta(2)^{5}}{\zeta(4)^{2}} M_{22}+O\left((\log q)^{5} \log _{2} q\right)
$$

and

$$
\widetilde{M}_{3}=O\left((\log q)^{5}\right) .
$$

3.5. Contribution of the residual quadratic forms. We have to show that the quadratic forms $\widetilde{M}_{21}^{i, \mathbf{i}, \mathbf{j}, \mathbf{k}}$ for $i<3$ do not contribute to the main term. All the necessary arguments were given in the preceding sections. After having computed, in terms of the $y_{k}$ 's, new variables of the form

$$
y_{k}^{i, j_{4}, k_{4}}:=\sum_{m} h^{j_{4}, k_{4}}(m)(\log m)^{i} x_{k m},
$$

one can show by cumbersome but easy calculations that for the mollifier chosen in (86) and (87), (82) holds for $i<3$.

3.6. Conclusion. From (91), (92), (88), and (74), we see that for $\Delta<1 / 4$ one has

$$
\sum_{\substack{f \in S_{2}(q)^{*} \\ L(f, 1 / 2)=0, L^{\prime}(f, 1 / 2) \neq 0}} 1 \geq \frac{\left(M_{1}^{\mathrm{n}}\right)^{2}}{M_{2}^{\mathrm{n}}}=q \frac{\zeta(2)}{2 \pi^{2}} \cdot \frac{M_{1}(\Delta)^{2}}{M_{2}(\Delta)}+O\left(\frac{\log _{2} q}{\log q}\right),
$$

and Theorem 3 follows (in a more precise form) since we know that

$$
q \frac{\zeta(2)}{2 \pi^{2}}=\frac{q}{12}=\operatorname{dim} J_{0}(q)+O(1) .
$$

In the case of the central critical values, we go from harmonic averages $N_{1}$ and $N_{2}$ to the natural averages, and prove Theorem 2 in a similar way. All computations being simpler, we omit the details.

\section{References}

[CGG] J. B. Conrey, A. Ghosh and S. M. Gonek, Simple zeros of the Riemann zeta function, Proc. London Math. Soc. (3) 76 (1998), 497-522.

[Du] W. Duke, The critical order of vanishing of automorphic L-functions with high level, Invent. Math. 119 (1995), 165-174. 
[DFI] W. Duke, J. Friedlander and H. Iwaniec, Bounds for automorphic L-functions, II, ibid. 115 (1994), 219-239.

[GR] I. S. Gradshteyn and I. M. Ryzhik, Table of Integrals, Series, and Products, 5th ed., Academic Press, 1994.

[GZ] B. Gross and D. Zagier, Heegner points and derivatives of L-series II, Math. Ann. 278 (1987), 497-562.

[Iw] H. Iwaniec, On the order of vanishing of modular L-functions at the critical point, Sém. Théor. Nombres Bordeaux 2 (1990), 365-376.

[ILS] H. Iw aniec, W. Luo and P. Sarnak, Low lying zeros for families of L-functions, preprint, 1998.

[IS] H. Iw aniec and P. Sarnak, The non-vanishing of central values of automorphic L-functions and Siegel's zeros, preprint, 1997.

[Jut] M. Jutila, Lectures on a Method in the Theory of Exponential Sums, Tata Lectures on Math. and Phys. 80, Springer, 1987.

[KS] N. Katz and P. Sarnak, Random Matrices, Frobenius Eigenvalues, and Monodromy, Amer. Math. Soc. Colloq. Publ. 45, 1999.

[Kow] E. Kowalski, The rank of the jacobian of modular curves: analytic methods, thesis, Rutgers University, May 1998 (available at http://www.princeton.edu/ekowalsk/These/these.html).

[KM1] E. Kowalski and P. Michel, The analytic rank of $J_{0}(q)$ and zeros of automorphic L-functions, Duke Math. J. 100 (1999), 503-542.

[KM2] - - - Explicit upper bound for the rank of $J_{0}(q)$, preprint, Université Montpellier II, 1998.

[Miy] T. Miyake, Modular Forms, Springer, 1989.

[Sh] G. Shimura, Introduction to the Arithmetic Theory of Automorphic Functions, Iwanami Shoten and Princeton Univ. Press, 1971.

[Sou] K. Soundararajan, Non-vanishing of quadratic Dirichlet L-functions at $s=$ $1 / 2$, preprint, 1999.

[VdK] J. VanderKam, The rank of quotients of $J_{0}(N)$, preprint, 1997.

Math. Dept.-Fine Hall

Washington Road

Princeton, NJ 08544-1000, U.S.A.

E-mail: ekowalsk@math.princeton.edu
Mathématiques, cc 051 Université Montpellier II Place Eugène Bataillon 34095 Montpellier Cedex 5, France E-mail: michel@darboux.math.univ-montp2.fr 